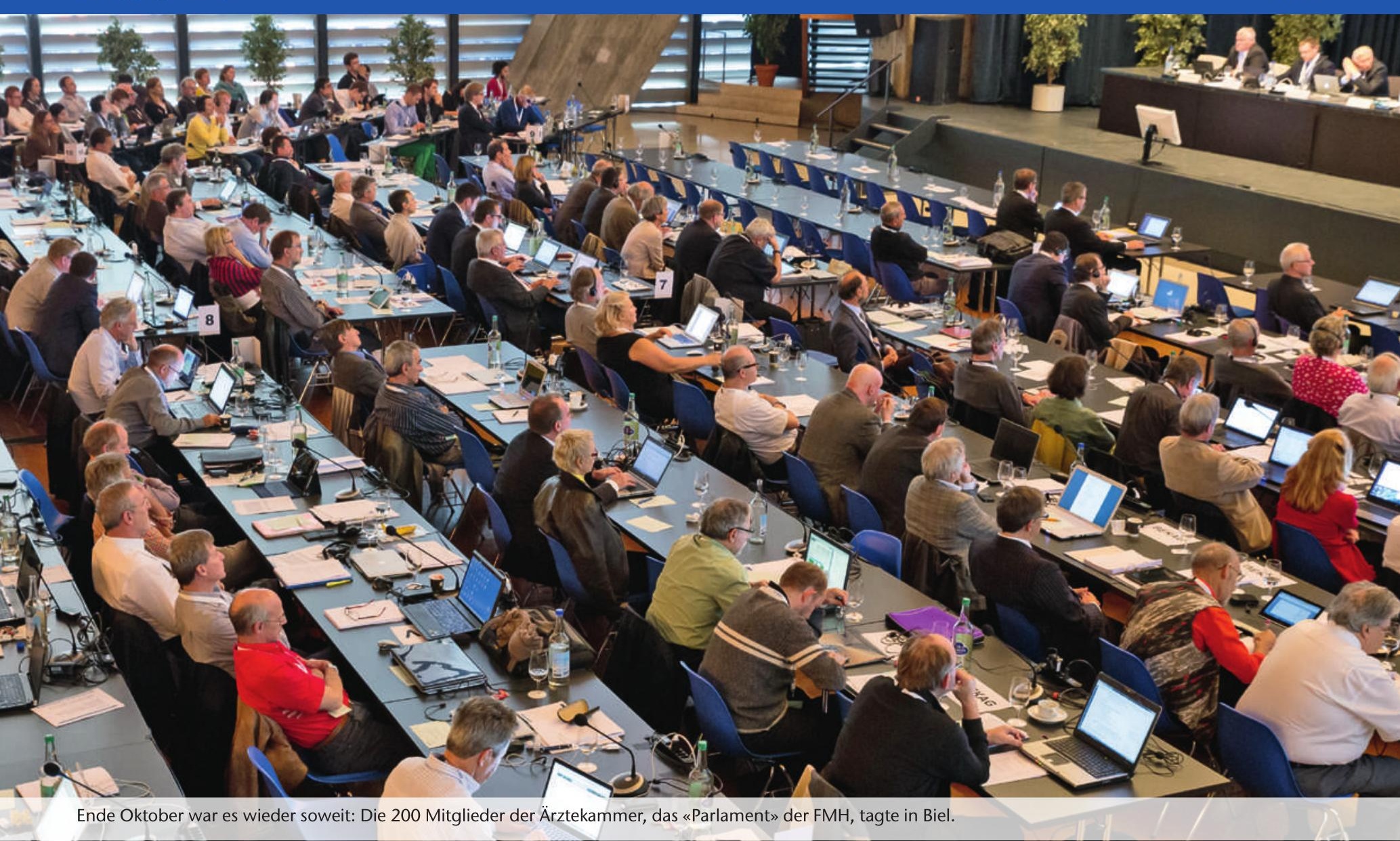

Kongresshaus Biel, 30. Oktober 2014

\title{
Protokoll der zweiten Ärztekammer im 2014
}

Monika Henzen

Leiterin Zentrales Sekretariat

Ein Glossar mit den am häufigsten verwendeten Abkürzungen finden Sie auf Seite 1953

\section{Begrüssung, Mitteilungen, Bestellung des Büros}

Jürg Schlup/Präsident FMH begrüsst die Delegierten zur zweiten Ärztekammer 2014. Als Gäste heisst er Joachim Eder/Ständerat, Hans-Ruedi Koelz/Vizepräsident SIWF, Bruno Kesseli/Chefredaktor SÄZ sowie René Häller/ Geschäftsführer FMH Consulting Services willkommen. Zu Traktandum 4 wird Christian Peier/Geschäftsführer IPI erwartet.

Anne-Geneviève Bütikofer/GS FMH gibt die üblichen organisatorischen Mitteilungen und verweist adie heute zusätzlich verteilten Sitzungsunterlagen. Sie informiert über das neu eingeführte Dokumentenmanagement-System. Ziel ist eine vereinfachte Verteilung und Zurverfügungstellung von Dokumenten für Organe, Gremien, Kommissionen und Projektgruppen. Der Versand per CD-ROM oder E-Mail fällt in Zukunft weg. Die Unterlagen für die nächste ÄK werden nicht mehr per E-Mail, sondern über das neue ITTool verschickt. Eine entsprechende Anleitung ist in den heute verteilten Unterlagen vorhanden.

Anschliessend bestellt der Präsident das Büro der ÄK. Dieses besteht aus dem Präsidenten, den Vizepräsidenten, der Generalsekretärin und den nachfolgenden Stimmenzählenden:
Pierre Klauser, Christoph Ramstein, Franziska Zogg, Marcel Stampfli, Jean-Michel Gaspoz, Christiane Roth, Josef Widler, Christoph Lüssi, Karl Lövblad, Fabrice Dami.

Die Stimmenzählenden werden ohne Gegenstimme gewählt.

\section{Traktandenliste}

Jürg Schlup/Präsident FMH hält fest, dass die Traktandenliste ordnungsgemäss zugestellt wurde. Rechtzeitig eingegangen ist der Ordnungsantrag der fmCh. Fünf weitere Anträge, welche heute als Tischvorlage aufliegen, sind nach dem Nachversand eingereicht worden.

Urban Laffer/Präsident fmCh begründet den Ordnungsantrag. Aufgrund deren Bedeutung und Dringlichkeit beantragt er, dass Traktandum 6 vor Traktandum 3 vorzuziehen sei und dass innerhalb des Traktandums 6 Traktandum 6.5 als erstes behandelt wird.

Jürg Schlup/Präsident FMH verdankt den Antrag und informiert, dass der ZV diesen ablehnt. Bei Genehmigung des Antrages der fmCh müsste die ÄK zuerst über Budgetmassnahmen beraten, die das Jahr 2016 betreffen. Die budgetrelevanten Anträge 2015 


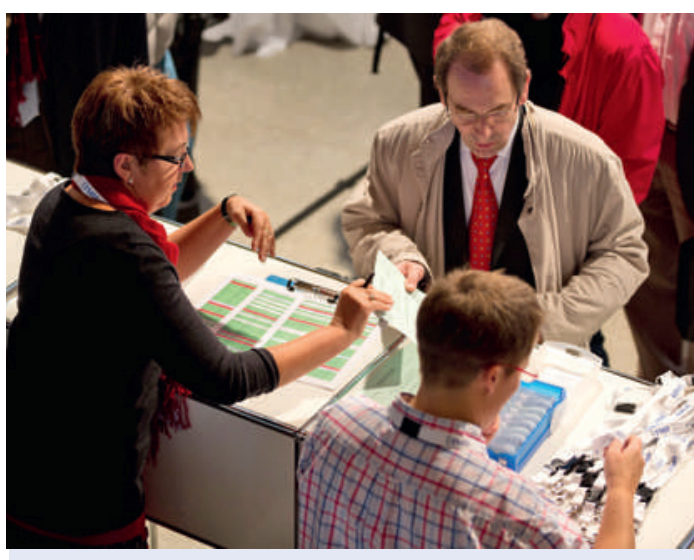

FMH-Mitarbeitende in Aktion: Die Delegierten erhalten ihren Badge und die Stimmunterlagen.

würden somit erst anschliessend diskutiert. Eine neue Reihenfolge der Budgettraktanden würde zu Unstimmigkeiten führen.

Adrian Sury/Präsident GPK spricht sich ebenfalls gegen den Ordnungsantrag der fmCh aus und unterstützt den Antrag des ZV. Bei dem von der GPK eingereichten Antrag betreffend Budgetstabilisierungsmassnahmen (Trakt. 6.5) geht es um die mittel- und langfristige Finanzplanung, welche erst 2016 wirksam wird.

Es wird über den Ordnungsantrag des fmCh abgestimmt:

Antrag fmCh:

Die fmCh beantragt, dass Traktandum 6 den Traktanden 3 und 4 vorgezogen wird und dass Traktandum 6.5 «Massnahmen zur Budgetstabilisierung» den Traktanden 6.1 bis 6.4 vorgezogen wird.

Beschluss:

Der Antrag fmCh wird mit $43 \mathrm{Ja}$ zu 75 Nein und 11 Enthaltungen abgelehnt.

Die ÄK stimmt über einen weiteren von Angelo Cannova/Ersatzdelegierter AGZ eingereichten Antrag ab, der jedoch von der AGZ nicht unterstützt wird. Angelo Cannova erhält die Gelegenheit, diesen zu präsentieren. Jürg Schlup/Präsident FMH dankt für die Ausführungen und informiert, dass der ZV diesen Antrag ebenfalls ablehnt.

\section{Antrag Angelo Cannova:}

1. In der Ärztekammer sollen die Reformprojekte im Gesundheitswesen wie «Interprofessionalität» und «neue Versorgungsmodelle für die medizinische Grundversorgung» eingehend dargelegt werden.

2. Es soll eine Kommission gebildet werden, die herausarbeitet und verständlich darlegt, in welche Gesetze diese Umgestaltungspläne einfliessen.

3. Über dieses Ergebnis soll in der kommenden Ärztekammer debattiert werden.
Beschluss:

Der Antrag Cannova wird mit 11 Ja zu 115 Nein und 6 Enthaltungen abgelehnt.

Die ordentlich zugestellte Traktandenliste wird somit ohne zusätzliche Traktanden genehmigt.

Der Präsident schlägt gemäss Art. 11 Abs. 3 der GO vor, dass ab 17.00 Uhr keine Beschlüsse mehr gefasst und keine Wahlen mehr durchgeführt werden.

Der Antrag wird mit 1 Gegenstimme und 1 Enthaltung gutgeheissen.

\section{Eröffnungsreferat des Präsidenten FMH}

In seinen Eröffnungsworten geht der Präsident auf die wichtigsten strategischen und politischen Schwerpunkte ein. Neben der Überprüfung der von der ÄK 2013 verabschiedeten Strategie der FMH für die Legislatur bis 2016 gab es 2014 viele gesundheitspolitische Geschäfte, bei der sich die FMH stark engagierte. Die Volksinitiative «Für eine öffentliche Krankenkasse» wurde mit 38\% Ja zu 62\% Nein verworfen. Der JA-Stimmenanteil hat gegenüber der Abstimmung 2007 zugenommen. Gemäss den offiziellen Stellungnahmen der vier Bundesratsparteien am Abend des Abstimmungstages fordern deren drei die Einschränkung der freien Arztwahl. Die FMH hat bereits vor der Abstimmung agiert und in einer Medienmitteilung eine Einschränkung der freien Arztwahl klar abgelehnt. Nach der Zustimmung des Nationalrates zu einer Einschränkung wird der Ständerat voraussichtlich in der Wintersession dieses Geschäft beraten. Die FMH wird ihre Ideen und Anliegen im Parlament einbringen und für ihre Interessen lobbyieren. Dabei ist sie auf die Unterstützung der angeschlossenen Ärzteorganisationen angewiesen. Sollte der Ständerat diesem Vorstoss ebenfalls zustimmen, erhält der Bundesrat den Auftrag, ab einer bestimmten Ärztedichte die Vertragsfreiheit einzuführen und dem Parlament die notwendigen Gesetzesänderungen vorzuschlagen. Die FMH hat zu den bedeutenden Vernehmlassungen Zentrum für Qualität in der obligatorischen Krankenversicherung und Steuerung im ambulanten Bereich ihre Stellungnahme beim Bundesrat eingereicht. Die FMH wurde zu den Hearings zum Heilmittelgesetz (SGK SR) und zum elektronischen Patientendossier (SGKNR) ebenfalls eingeladen. Zwei Parlamentarieranlässe zu Interprofessionalität und Vertragsfreiheit sowie zum MedBG (Sprachtest) wurden durchgeführt.

Eine Umfrage von Robert J. Blendo zum Thema «Public Trust in Physicians - U.S. Medcine in international Perspective», publiziert im NEJM am 23. Oktober 2014, attestiert der Ärzteschaft in der Schweiz das höchste Vertrauen der Bevölkerung von allen untersuchten 29 Industrieländern. Dies ist nicht selbstverständlich. Heute stehen die Ärzte einer überbordenden administrativen Kontrolle und einer Misstrauenskultur seitens der Versicherer gegenüber, die 
demotiviert. Es braucht jedoch eine vertrauensvolle Zusammenarbeit und gegenseitige Verständigung mit allen Anspruchsgruppen im Gesundheitswesen. Die Ärzteschaft gerät zunehmend in eine mission impossible, angesichts umfassender Leistungsversprechen der Krankenkassen bei gleichzeitigen Sparvorgaben. Es kann nicht Unmögliches geleistet werden. Die Ärzte wollen dazu beitragen, eine qualitativ hochstehende und gute Versorgung sicherzustellen, aber das wird kostenneutral nicht möglich sein. Heute wird eine Behörde geschaffen, die medizinische Qualität verwaltet. Die Ärzteschaft ist gegen solche Bestrebung. Sie will darin unterstützt werden, Qualität zu produzieren und zu verbessern. Dazu braucht es ein Bundesamt mit ärztlichem Sachverstand in der Geschäftsleitung.

Der Präsident dankt an dieser Stelle allen Kolleginnen und Kollegen für ihre tägliche Arbeit zum Wohl der Patientinnen und Patienten.

\section{2. Änderungen in der Geschäftsordnung sowie in der Standesordnung}

2.1 Anpassung Geschäftsordnung «Übersetzungen in der DV»: Anpassung Art. 18, Abs. 2 und Art. 19 Abs. 2

Die DV wünscht eine Übersetzung einzelner Rubriken auf dem Rundschreibeformular sowie eine mündliche Übersetzung während den Sitzungen. Hanspeter Kuhn/ FMH erläutert die Ausgangslage sowie den Antrag. Eine Zustimmung hat eine Änderung der GO zur Folge.

\section{Antrag:}

Revision von Art. 18 Abs. 2 GO

Anträge auf Traktandierung eines Geschäfts werden mittels Rundschreiben zuhanden des GS eingereicht. Sie enthalten unter anderem den Sachverhalt, eine Umschreibung des Problems und einen Beschlussvorschlag. Die Rubriken «Zusammenfassung» und «Beschluss» des Rundschreibeformulars werden bei Eintreffen bis zwei Wochen vor Versand der Einladung vom GS und bei späterem Eintreffen vom Autor des RS nicht übersetzt.

Revision von Art. 19 Abs. 2 GO:

Die Verhandlungen der DV sind nicht öffentlich. Sie und werden mündlich nicht simultanübersetzt.

Beschluss:

Dem Antrag wird ohne Gegenstimme und mit 1 Enthaltung zugestimmt.

\subsection{Ergänzung der FMH-Standesordnung}

\subsubsection{Aufnahme SAMW-Richtlinien} «Abgrenzung von Standardtherapie und experimenteller Therapie im Einzelfall» Im Mai 2014 hat die SAMW die neuen medizinischethischen Richtlinien und Empfehlungen «Abgrenzung von Standardtherapie und experimenteller Therapie im Einzelfall» beschlossen. Hanspeter Kuhn/FMH hält fest, dass das Humanforschungsgesetz nur die systematische Forschung regelt. Nicht im Gesetz geregelt wird richtigerweise die experimenteller Therapie im Einzelfall. Die Richtlinie der SAMW schliesst die vom Gesetzgeber bewusst offengelassene Lücke durch Selbstregulierung der medizinischen Institutionen. Es handelt sich um folgende Ergänzung in der FMH-Standesordnung:

Art. 18 Richtlinien der Schweizerischen Akademie der Medizinischen Wissenschaften (Ergänzung)

Bezüglich [...] Abgrenzung von Standardtherapie und experimenteller Therapie im Einzelfall gelten die Richtlinien der Schweizerischen Akademie der Medizinischen Wissenschaften [...]

Beat Gafner/BE kann nicht nachvollziehen, warum die SAMW die von der BEKAG im Rahmen des Vernehmlassungsverfahrens eingegebene Stellungnahme bezüglich Möglichkeit der vorgängigen Rücksprache mit nahen Angehörigen und Bezugspersonen nicht berücksichtigt hat. Er stellt weiter die Frage, ob das erwähnte Austauschprinzip über die Kostenregelung bei experimentellen Therapien realistisch eingeschätzt wird?

Hanspeter Kuhn/FMH meint, dass für den ersten Punkt bei der SAMW Rücksprache genommen werden muss. Bei der Kostenregelung hingegen besteht beim Bundesgericht die ständige Praxis, bei stationären Behandlungen das Kostenäquivalenzprinzip anzuwenden. Für den Bereich der ambulanten Medizin ist kein Bundesgerichtsentscheid bekannt, der diese Haltung stützt. Die Frage ist, wie die Ärzteschaft damit umgehen soll. Nach seinem Verständnis wäre es ein sinnvolles Zeichen, wenn die ÄK dieser Richtlinie zustimmt, weil ja das Parlament private Regelungen in Aussicht gestellt hat.

Jürg Nadig/SGMO weist seine Kolleginnen und Kollegen darauf hin, dass mit dieser Regelung der Patient für jeden off-label use sein Einverständnis geben muss. Für ihn ist das erläuternde Schema irreführend, da es nicht mit dem Text übereinstimmt. Bei der Tabelle wird aufgeführt, dass beim off-label use nur die Kosten der Medikamente übernommen werden. Das ist jedoch nicht der Bereich, der zur Diskussion steht. Es stellt den Antrag, die Richtlinie nochmals mit der SAMW zu besprechen und das Geschäft auf eine nächste ÄK zu verschieben.

\section{Antrag Jürg Nadig:}

Die offenen Fragen der Richtlinie werden mit der SAMW nochmals besprochen. Das Geschäft wird auf eine nächste ÄK verschoben.

\section{Beschluss:}

Der Antrag wird mit 125 Ja zu 10 Nein und 17 Enthaltungen angenommen.

Jürg Schlup/Präsident FMH bittet die beiden Votanten, ihm die entsprechenden Anträge für die Verhandlungen mit der SAMW einzureichen. 


\subsubsection{Neuer Art. 22bis}

\section{«Gleichbehandlung Werbung»}

Die DV hat im Mai 2014 die Situation betreffend Werberecht für Ärztinnen und Ärzte im heutigen Umfeld diskutiert. Hanspeter Kuhn/FMH informiert über die Resultate der von der DV eingesetzten Arbeitsgruppe. Diese schlägt vor, sich in einer Ergänzung der Standesordnung auf das Anliegen der Gleichbehandlung der Werbung von Ärztinnen und Ärzten und anderen Akteuren (Spitäler, Versicherer) in der Region zu konzentrieren. Ziel ist nicht eine Lockerung des FMHStandesrechts, sondern die Aufforderung an die Behörden, bei den nicht der Standesordnung unterstehenden Akteuren Grenzen zu setzen.

Anja Zyska/VSAO möchte wissen, was bei Annahme dieser Richtlinie an der aktuellen Praxiswerbung bei Krankenhäusern und Behandlungsinstituten ändert. Dürfen diese demnach Werbung machen mit dem Hinweis, Arzt so und so bietet das und das an?

Hanspeter Kuhn/FMH hält fest, dass die Frage der Werbung im kantonalen Spitalgesetz geregelt ist. Es gibt keine einheitliche schweizerische Regelung. Die Ärzteschaft kann im Standesrecht die Werbung ihrer Mitglieder regeln. Wer der FMH nicht beitreten will, für den gilt das nicht. Die Spitäler stehen einzig unter Aufsicht der kantonalen Gesundheitsdirektoren. Es geht hier darum, dass die Ärzteschaft in der Region entsprechend der kantonalen Regelung und Praxis ihre eigenen Werbeaktivitäten gestalten kann, damit die praktizierenden Ärzte im Konkurrenzkampf mit anderen Anbietern nicht benachteiligt sind.

Auf die Frage von Ricardo Torriani/ZH, ob er machen könne, was er wolle, antwortet Hanspeter Kuhn/FMH, dass de facto die Standeskommission im Kanton Zürich bei ihm so viel zulassen wird, wie im Moment bei den Spitälern zugelassen ist. Das, was die anderen ungehindert tun können, könne auch er tun.

Hans Ulrich Iselin/AG hält dies für eine unbefriedigende Lösung. Wenn die Standesregeln so eng belassen werden, wie bis anhin, bringt dieser Vorschlag

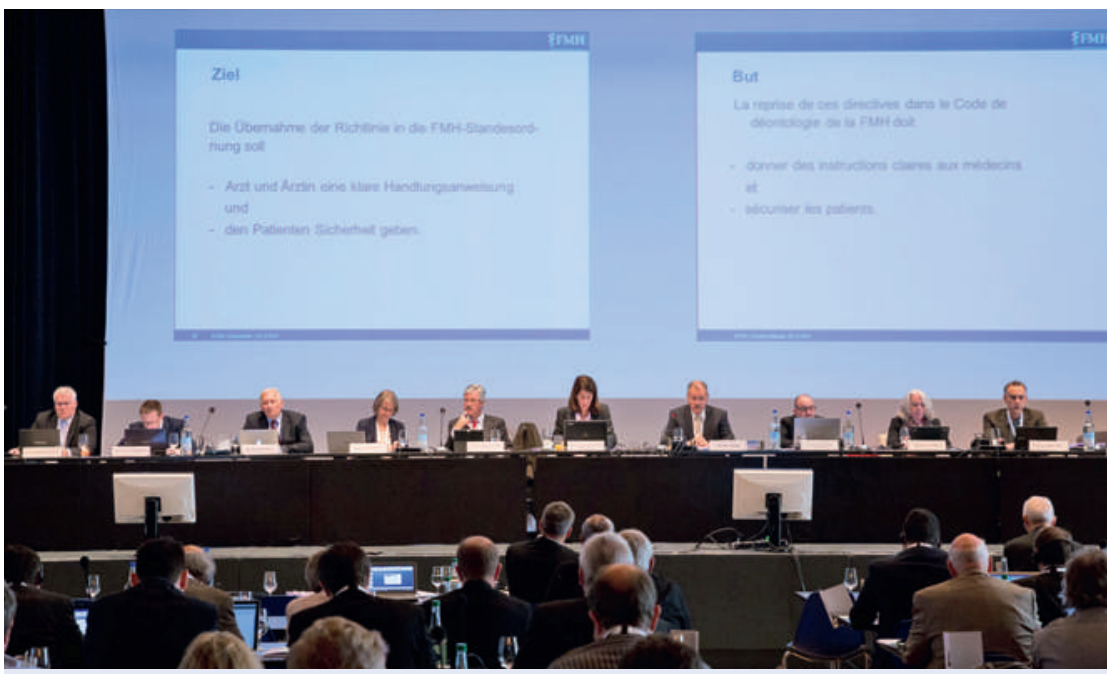

Der Zentralvorstand und die Generalsekretärin verfolgen die Diskussion im Plenum. nichts. Die Standesregeln sind so anzupassen, dass privat praktizierende Ärzte die gleiche Werbemöglichkeit haben wie grössere Institutionen.

Hanspeter Kuhn/FMH versteht das Votum für eine grössere Liberalisierung. Das ist jedoch eine politische und keine rechtliche Entscheidung. Die Ärzteschaft muss für sich überlegen, was der richtige Weg in die Zukunft sein soll. Die Arbeitsgruppe hat die vorgeschlagene Regelung als zentrales Anliegen erachtet.

Für Marc Müller/Hausärzte Schweiz fordert diese Regelung die Regulatoren auf, auch bei den Spitälern engere Grenzen zu setzen. Er zweifelt daran, ob die Regulatoren diesen Wink mit dem Zaunpfahl verstehen und ob mit dieser Regelung nicht ein Chaos ausgelöst wird.

Antrag Ergänzung der FMH-Standesordnung: Art. 22b (neu) Regionale Gleichbehandlung mit Spitälern und anderen Behandlungsinstitutionen

Die Standeskommission gewährleistet bei der Durchsetzung der Art. 19, 20 und 22 sowie der Anhänge 2 und 3 zur Standesordnung (FMH Richtlinien «Information und Werbung» und «Richtlinien für die Medientätigkeit von Ärztinnen und Ärzten») die Gleichbehandlung von Arzt und Ärztin mit Spitälern und anderen Behandlungsinstitutionen in derselben Region, indem sie die Praxis der kantonalen und eidgenössischen Aufsichtsbehörden betreffend deren Werbung bzw. der Werbung von Dritten für diese berücksichtigt.

\section{Beschluss:}

Der Antrag wird mit 124 Ja zu 17 Nein und 9 Enthaltungen angenommen.

\section{Sitzungs- und Spesenentschädigungen für FMH-Organe und Kommissionen}

Anne-Geneviève Bütikofer/GS FMH erläutert, dass die bisherige Regelung der FMH zum Teil lückenhaft, intransparent und schwer nachvollziehbar ist. Sie weist Ungleichbehandlungen auf und ist mit einem latenten Steuerrisiko verbunden. Die Ausgleichskasse hat bei der letzten Revision eine Änderung verlangt. Der ZV hat verschiedene Varianten ausgearbeitet, welche an der DV vom 22. Mai 2014 und 25. Juni 2014 eingehend diskutiert wurden. Es soll anstelle der bisherigen ganz- und halbtägigen Entschädigungen für sämtliche Organe und Kommissionen neu eine Entschädigung auf Basis eines Stundensatzes von CHF 170 eingeführt werden. Die DV hat einen zusätzlichen Vorschlag ausgearbeitet. Sie bevorzugt die Variante mit einer Entschädigung der ÄK-Delegierten durch die delegierenden Gesellschaften, bei welcher diese die Höhe der Entschädigung selber bestimmen. Sie hat sowohl dem Vorschlag des ZV wie auch dem eigenen zugestimmt und einstimmig beschlossen, der ÄK beide Varianten zu unterbreiten. Die Mühewalt der Mitglieder der GPK soll beibehalten werden.

Ricardo Torriani/ZH spricht sich aus strategischen Gründen für einen einheitlichen Satz von CHF 170 
für alle aus. Es geht auch künftig darum, engagierte Mitglieder in der ÄK zu haben. Eine Einsparung findet er eine schlechte Investition in die Zukunft.

Peter Wiedersheim/SG, Präsident DV argumentiert, dass der Antrag um Übernahme der Kosten durch die delegierenden Gesellschaften aus den Kantonen kommt. Der ZV bevorzugt eine einheitliche Entschädigung. Da die delegierenden Gesellschaften bereits über alle Daten verfügen, würde der Vorschlag des ZV der FMH einen zusätzlichen administrativen Aufwand bringen. Die DV jedoch will mit ihrem Vorschlag weitere administrative Kosten einsparen. Das Budget sollte entlastet und nicht zusätzlich belastet werden.

Daniel Schröpfer/VSAO erklärt, dass die GPK die heutige Entschädigungsregelung der Reisespesen gerügt hat, da diese als versteckte Lohnkosten beurteilt werden. Der VSAO plädiert für eine Entschädigung durch die delegierenden Fachgesellschaften. Er befürwortet einen einheitlichen Stundenansatz, wobei bei einem Ansatz von CHF 170 über die Ausgewogenheit diskutiert werden kann.

Monique Lekhy Hagen/VS begrüsst den Vorschlag, dass die delegierenden Gesellschaften die Kosten übernehmen. Um das Budget der FMH jedoch wirksam zu entlasten, sollten die DV-Delegierten ebenfalls durch die delegierenden Gesellschaften entschädigt werden. Diese Regelung würde zudem vielleicht auch dazu beitragen, den Informationsfluss innerhalb der Fachgesellschaften zu verbessern. Sie stellt einen entsprechenden Antrag.

Vincenzo Liguori/TI unterstützt diesen Antrag aus Gründen der Transparenz und einer Entlastung des Budgets. Was den Stundenansatz von CHF 170 betrifft, vertritt er die Meinung, dass dieser nicht mit dem TARMED zu vergleichen ist, da die Arbeitsstunden in einem Gremium nicht mit jenen im Praxisalltag gleichzustellen sind.

Ralph Litchel/VSAO wünscht eine genaue Kostenübersicht der beiden Varianten «Einheitliche Entschädigung mit ÄK-Delegierten» und «Einheitliche Entschädigung ohne ÄK-Delegierte».

Anne-Geneviève Bütikofer/GS FMH führt aus, dass der Vorschlag des ZV mit einem einheitlichen Stundenansatz inkl. ÄK-Delegierte eine Ausgabenerhöhung von CHF 360000 mit sich bringt. Der Vorschlag der DV mit einem einheitlichen Stundenansatz ohne ÄK-Delegierte entspricht einer Ausgabenreduktion von CHF 40000.

Franziska Zogg/ZG stellt klar, dass in der Stundenentschädigung der DV-Delegierten von CHF 170 die Sitzungsvorbereitung ebenfalls inbegriffen ist. Die DV ist ein beratendes richtungsweisendes Organ für die FMH und hat eine andere Aufgabe als die ÄK. Als beratendes Organ der FMH sollte die DV auch von der FMH bezahlt werden.

Pierre Vallon/FMPP schliesst sich diesem Votum an. Die DV-Delegierten werden von den Dachverbänden nominiert und investieren sechsmal jährlich einen ganzen Sitzungstag. Er empfiehlt, den Antrag von Frau Lehky Hagen abzulehnen.

\section{Antrag Lehky Hagen:}

Die Abstimmung betreffend Entschädigung der DVDelegierten ist auf die nächste $\ddot{A K} \mathrm{zu}$ verschieben. Es soll eine dritte Variante zur Abstimmung gelangen, dass die DV-Delegierten ebenfalls von den delegierenden Gesellschaften entschädigt werden.

Beschluss:

Der Rückweisungsantrag wird mit 5 Ja zu 140 Nein und 5 Enthaltungen abgelehnt.

Nach Rückweisung des Antrages Lehky Hagen werden über die Anträge des ZV und der DV abgestimmt:

Antrag ZV: Einheitlicher Stundenansatz mit ÄK-Delegierten:

Einführung der neuen Sitzungs- und Spesenentschädigungen für alle FMH Organe und Kommissionen mit einem einheitlichen Stundensatz von CHF $170 \mathrm{ab}$ 1.1.2015.

Antrag DV: Einheitlicher Stundenansatz ohne ÄKDelegierte:

Einführung der neuen Sitzungs- und Spesenentschädigungen ohne ÄK-Delegierte. Die Entschädigung der ÄK-Delegierten wird durch die delegierende Gesellschaft geregelt. Diese bestimmen die Höhe der Entschädigung.

\section{Beschluss:}

Für den Antrag der DV stimmen 134, für den Antrag des ZV 24, Enthaltungen 4. Der Antrag der DV wird somit mit 134 Stimmen gegen 24 Stimmen angenommen. Die Entschädigung der ÄK-Delegierten wird künftig durch die delegierende Gesellschaft übernommen. Diese bestimmen die Höhe der Entschädigung. Die Mühewalt zugunsten der Mitglieder der Geschäftsprüfungskommission ist beizubehalten.

\section{Institut für Praxisinformatik IPI}

- Jahreszwischenbericht des ZV betreffend Leistungsvertrag zwischen FMH und IPI

- Verlängerung der finanziellen Unterstützung und des Leistungsvertrages FMH - IPI

Anne-Geneviève Bütikofer/GS FMH präsentiert den Jahreszwischenbericht des ZV betreffend Leistungsvertrag (LV) zwischen FMH und IPI. Sie erinnert daran, dass die DV im Mai 2013 die Zusammenarbeit zwischen der FMH und dem IPI vorgeschlagen und der Variante mit Beginn auf Mandatsbasis und Evaluation für eine zukünftige Integration des IPI in das Departement eHealth genehmigt hat. Die AG eHealth hat an der DV im Juni 2013 einen Mandats- und Finanzierungsplan präsentiert und drei Projekte für den Beginn empfohlen. Das IPI hat an der DV im September 
2013 die Übertragung des Gesamtmandats und Finanzierung über einen Sonderbeitrag von CHF 30 beantragt. Die ÄK hat im Oktober 2013 diesem Sonderbeitrag zugestimmt.

Der LV 2014 wurde mit dem IPI im Februar 2014 unterzeichnet. Darin wurden neben 17 Projekten auch die Vorlage eines Business- und Finanzplans für 2014 und Folgejahr 2015-2017 definiert. Der Projekterfolg sollte anhand einer Balanced Score Card gemessen werden. Zur Überwachung und Begleitung der Verpflichtungen wurde ein Lenkungsausschuss (LA) eingesetzt, der paritätisch (3 FMH, 3 IPI) zusammengesetzt ist. Der LA hat 2014 viermal getagt.

Der vorliegende Jahreszwischenbericht wurde vom ZV im August 2014 und von der DV im September 2014 genehmigt. Gemäss Bericht diente das Geschäftsjahr 2014 unter anderem dem Aufbau der Geschäftsstelle sowie der Erarbeitung der Aufbau- und Ablauforganisation des IPI. Mit der Einstellung des Geschäftsführers per 1. März 2014 konnten nach Aussagen des IPI alle Projekte und Entwicklungsgrundlagenarbeiten initiiert werden. Die unerwartet lange Aufbauarbeit für die Geschäftsstelle hat bei den Projekten und Dienstleistungen zu Verzögerungen geführt. Der bisherige Verlauf der Projektarbeiten lässt darauf schliessen, dass die Anzahl der vom IPI festgelegten Projekte zu ambitioniert war. Angesichts der Ressourcenproblematik und der nicht umgesetzten Projektkontrollmechanismen besteht die Gefahr, dass der LV, welchen die ÄK auf Mandatsbasis beschlossen hat, nicht abschliessend erfüllt werden kann. Nicht abgeschlossene Projekte müssen auf 2015 übertragen und die Finanzhilfe (Vorauszahlungen) muss zurückgefordert werden. Der Nachweis der verwendeten Mittel für die definitive Zahlung ist noch zu erbringen. Das Projektbudget kann nicht ins nächste Jahr übertragen werden.

Anne-Geneviève Bütikofer/GS FMH unterstreicht die gute Zusammenarbeit zwischen dem IPI und der FMH durch den LA und das Departement eHealth mit der AG eHealth sowie das grosse Engagement des Geschäftsführers, Christian Peier. Der ZV empfiehlt die Weiterführung der finanziellen Unterstützung für 2015 nach der Unterschrift des Nachtrages zum LV und dessen Anhängen.

Beat Gafner/BE hat bis heute noch nie einen solch stringenten LV gesehen. Die Startpiste für das IPI musste wohl korrigiert werden, aber das Flugzeug hebt nun ab. Der Bedarf ist nachgewiesen und es wäre falsch, den LV nicht zu verlängern.

Daniel Schröpfer/VSAO findet die Idee des IPI gut. Nach den heutigen Ausführungen und dem Bericht des IPI zuhanden der DV im September 2014 ist es für den VSAO bedenklich, dem LV nochmals zuzustimmen. Sie lehnen eine Verlängerung ab.

Gerhard Schilling/Präsident IPI ersucht, das Wort Christian Peier, Geschäftsführer IPI, zu erteilen.

Christian Peier/Geschäftsführer IPI bedankt sich für die Möglichkeit, seine Sicht darzulegen. Seit seinem
Amtsantritt im März 2014 hat er die Projektliste übernommen und das IPI neu strukturiert. Er präsentiert eine Übersicht der Projekte und der bereits erreichten Resultate. Christian Peier ist überzeugt, weitere Meilensteine bis Ende 2014 erreichen zu können. Die Zusammenarbeit in der Industrie wird sehr geschätzt. Mit verschiedenen Partnern konnte zwischenzeitlich auch die Vereinbarung über einen gemeinsamen Standard betreffend Migrierbarkeit abgeschlossen werden. Das Bedürfnis ist vorhanden und es gibt aktuell keine Alternative. Er plädiert für die Unterstützung.

Marc Müller/Hausärzte Schweiz weist darauf hin, dass das EPD kommt. Bis heute jedoch fehlen Grundlagen und vernetzbare Programme, die eine Umsetzung in den Praxen ermöglichen. Es braucht einen einheitlichen Auftritt, um sich gegen die SoftwareIndustrie durchzusetzen. Das IPI hat vor sieben Monaten die operative Tätigkeit aufgenommen und ist auf Kurs. Es ist wahrscheinlich, dass bis Ende 2014 weitere Meilensteine erreicht werden können. Die Verlängerung des Mandats wie die Bewilligung des Sonderbeitrags sind unabdingbar. Bei Ablehnung wäre der Scherbenhaufen wie auch der Preis viel grösser und würde die Ärzteschaft um Jahre zurückwerfen. Er appelliert für eine Verlängerung dieses Mandates.

Gerhard Schilling/Präsident IPI hält fest, dass sich die Ärzteschaft bis heute kaum mit der komplexen Materie eHealth und IT sowie den Bedürfnissen beschäftigt hat. Die erlassenen Gesetze sind weder praxistauglich noch umsetzbar. Das Problem der Migrierbarkeit ist etwas ganz Zentrales. Der Umstieg auf eine elektronische Dokumentation kostet zwischen CHF 50000-100000. Und bei dieser Investition ist unsicher, ob die Firma in zwei Jahren noch existiert. In der Industrie wie in der Politik ist das IPI anerkannt. Die Zusammenarbeit mit namhaften Firmen funktioniert und es ist ausserordentlich wichtig, dass die Ärzteschaft ein verlässlicher Partner ist.

Anja Zyska/VSAO hält fest, dass der VSAO nicht wegen Geschäftsführungsmängeln gegen das IPI ist. Der VSAO unterstützt die Idee der Weiterführung der Projekte. Diese sollten jedoch nicht beim IPI, sondern beim Departement eHealth angesiedelt werden.

Sonja Trüstedt/VSAO pflichtet der Vorrednerin bei. Schon die Vorbereitungsphase hat zu Problemen geführt. Grosse Hindernisse stehen noch bevor und es ist ungewiss, wie diese überwunden werden können. Eine Verweigerung zur Weiterführung kann die FMH zurücksetzen. Aber oft ist es besser, rechtzeitig umzusteigen und später anzukommen.

Philippe Vuillemin/VD hätte einer Verlängerung um ein Jahr zugestimmt, auch im Wissen, dass die früher gesetzte Frist für die Erreichung der Meilensteine zu kurz war. Aber aufgrund der präsentierten Resultate sieht er sich verpflichtet, bereits dieses Jahr gegen den Antrag des ZV zu stimmen. Es handelt sich nun um einen wiederkehrenden Sonderbeitrag. Das nächste Jahr wird noch schwieriger. Der Sonderbeitrag wird von jedem Mitglied bezahlt. Deshalb sollte 


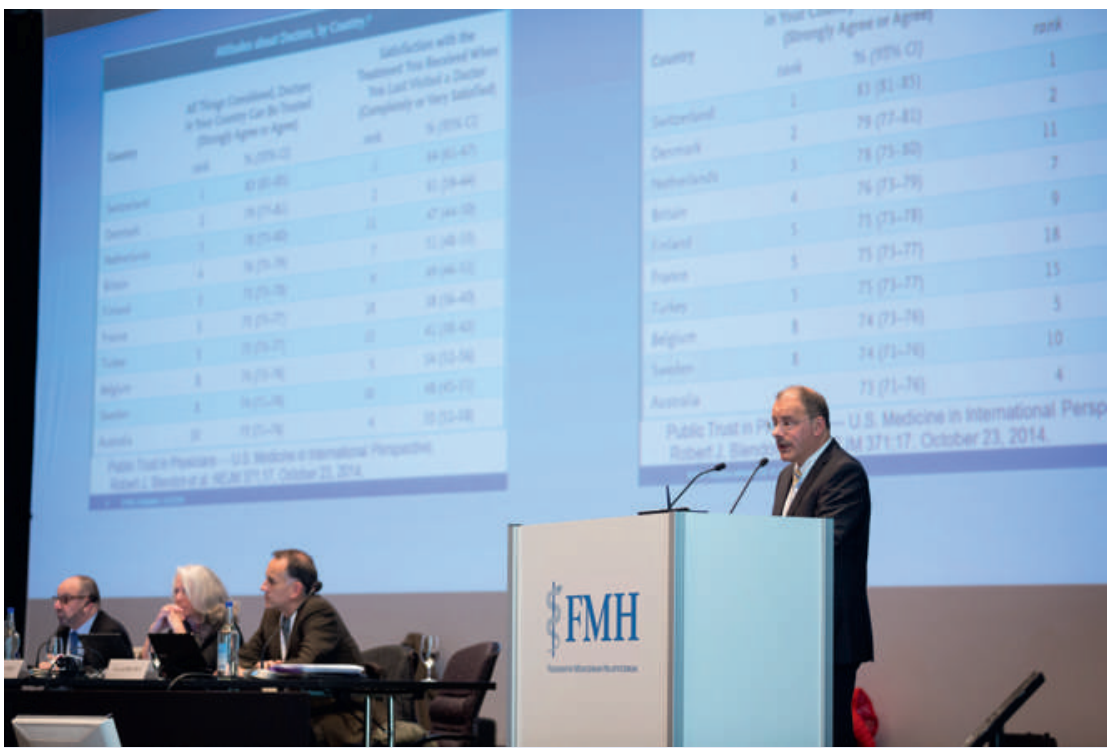

Der Präsident, Jürg Schlup, informierte über die wichtigsten gesundheitspolitischen Aktivitäten der FMH.
Hans-Jakob Riedtmann/ZH ist der Meinung, dass die ständig geforderten Sonderbeiträge in den Grundbetrag einzuberechnen seien. Die Erhebung von Sonderbeiträgen kann gegenüber den Mitgliedern nicht mehr länger vertreten werden.

Hanspeter Kuhn/FMH weist auf die Definition der Sonderbeiträge in Art. 11 der FMH-Statuten hin: «Die FMH und die KG setzen unter Beachtung des Kostenverursacherprinzips folgende Mitgliederbeiträge fest: - den allgemeinen jährlichen Grundbeitrag; - allfällige Sonderbeiträge für bestimmte Mitgliedergruppen oder für bestimmte Projekte. Die Höhe des Grundbeitrags bemisst sich nach der beruflichen Stellung des Mitglieds.»

Daniel Schröpfer/VSAO weist darauf hin, dass bereits vor zwei bis drei Jahren über die Integration des IPI ins Departement eHealth diskutiert wurde. Das Departement hat damals Vorschläge unterbreitet, aber eine Integration in die FMH wurde dannzumal nicht gewünscht. Und, das IPI ist mittlerweile über zehn Jahre alt.

Vincenzo Liguori/TI erinnert die heutige Debatte an jene aus dem Jahre 2009, wo es um die Einführung der HPC ging. Die FMH hat damals CHF 6 Mio. investiert und ist nach wie vor mit vielen Hindernissen konfrontiert. Alle sind für Verbesserungen des Systems, aber die FMH sollte aus den Fehlern der Vergangenheit lernen.

Jana Siroka/VSAO stellt den Antrag, dass bei Bewilligung des Sonderbeitrags von CHF 30 die Verantwortung dem Departement eHealth übertragen wird. Den Entscheid einer Integration soll nicht mehr das IPI, sondern das Departement eHealth fällen.

Die Anträge Nadig und Siroka bedingen als erstes die Abstimmung über den Antrag des ZV. und nicht mehr von der Integration in die FMH gesprochen.

Christian Peier/Geschäftsführer IPI erklärt, dass die Integration des IPI in die FMH gemäss Businessplan in den nächsten zwei Jahren vorbereitet wird.

Jürg Nadig/SGMO findet das IPI für die Ärzte zentral. Für den Fall, dass der Antrag des ZV abgelehnt wird, schlägt er als Alternative vor, die Integration des IPI in die FMH um ein Jahr vorzuziehen. Dieser Antrag gelangt nur zur Abstimmung, wenn jener des ZV abgelehnt wird.

Josef Widler/ZH appelliert für die Unterstützung des ZV-Antrags, da es zur Zeit keine Alternative gibt. Die praktizierenden Ärzte seien - im Vergleich zu den Spitalärzten - auf ein Vorwärtskommen angewiesen.

Urs Stoffel/ZV FMH führt aus, dass das Departement eHealth dieses Projekt seit Beginn begleitet und unterstützt hat. Die Zusammenarbeit mit dem Geschäftsführer IPI ist eng und dieser ist in der AG eHealth gut integriert. Aus Sicht des Departements ist die Aufgabe ausserordentlich wichtig. Die Ärzteschaft muss die Themen, die das IPI bearbeitet, auch in Zukunft ernst nehmen und einen Beitrag leisten. Es kann nicht sein, dass das IPI heute ohne jegliche Alternative begraben wird.

\section{Antrag ZV:}

Die ÄK nimmt den JahresZwischenbericht des ZV zur Kenntnis und verlängert den Leistungsvertrag zwischen der FMH und dem IPI einzig für ein Jahr.

\section{Beschluss: \\ Dem Antrag wird mit 88 Ja zu 65 Nein und 4 Enthal- tungen zugestimmt.}

Der Antrag Nadig wird mit dem Abstimmungsresultat gegenstandslos. Jana Siroka/VSAO zieht ihren Antrag zurück.

\section{Gastreferat}

Als Gastreferentin begrüsst Jürg Schlup/Präsident FMH Frau Prof. Dr. med. Dr. phil. Nikola Biller-Andorno. Sie ist seit 2005 ordentliche Professorin und leitet seit 2007 das Institut für Biomedizinische Ethik an der Universität Zürich.

In ihrem Referat «Patientenwohl und Ökonomie - Ärzte als Diener zweier Herren» geht Prof. Biller-Andorno auf das Spannungsfeld zwischen Patientenwohl und Ökonomie ein. In der Schweiz, die 
weltweit die zweithöchste Lebenserwartung hat, wo 87\% der Schweizer Bevölkerung ihren Gesundheitszustand mindestens als gut bezeichnet und die Mehrheit mit dem Gesundheitswesen zufrieden ist, bilden die Kosten den einzigen Wermutstropfen. Es ist völlig richtig, dass die Frage der Finanzierbarkeit oder «was ist ein angemessenes Kosten-Nutzen-Verhältnis» nicht objektiv bestimmbar sind, wie Jürg Schlup kürzlich in der SÄZ anmerkte. Das Gesundheitsbudget der Schweiz ist nicht in Stein gemeisselt, wir stossen immer mehr an eine Grenze der Bereitschaft, mehr für die Gesundheit auszugeben. Ökonomisches Denken in der Medizin ist im Sinne eines nachhaltigen Gesundheitssystems wünschenswert, darf sich jedoch nicht als Bedrohung für eine gute Medizin auswirken.

Diesem Spannungsfeld zwischen Medizin und Ökonomie hat sich das kürzlich publizierte Positionspapier der Schweizerischen Akademie der Medizinischen Wissenschaften (SAMW) zugewandt und beschreibt Problemfelder, die im klinischen Alltag im Zuge der Ökonomisierung auftreten (www.samw.ch/ de/Publikationen/Positionspapiere). Was dort eine Liste von sieben Punkten ist, findet sich in der Praxis eher als Geflecht verschiedener Problemstellungen, die im Positionspapier mit Hilfe verschiedener Vignetten abgebildet werden sollen. Diese zeigen auf, wie sich Prozesse der Effizienzsteigerung und Interessenkonflikte in Qualitätseinbussen, Über- oder Unterversorgung niederschlagen können und dabei das Fürsorgemodell aushöhlen und einer De-Professionalisierung Vorschub leisten können.

Die Wahrnehmung der Ärzteschaft kann als frühe Warnung dienen, wenn sich Mechanismen zur Effizienzsteigerung nachteilig auswirken. Da die Kosteneindämmung durch wirtschaftliche Anreize bei gleichzeitig qualitativ hochstehenden umfassenden Leistungsangeboten oberstes Ziel der DRG-Einführung waren, lohnt es sich, hier näher hinzuschauen. Trotz der Erfahrung, dass die Befürchtung «blutiger Entlassungen» unbestätigt blieb, könnte es durchaus sein, dass Anreizsysteme wie DRGs zu subtilen Qualitätsverlusten führen, die aber dennoch den Kern der Medizin betreffen.

In der im Sinergia-Programm des Nationalfonds durchgeführten Studie «Assessing the Impact of DRGs on Patient Care and Professional Practice» (IDoC) wurden landesweit Spitalärzte gefragt, wie sich ihr Berufsalltag unter den Fallpauschalen verändert hat. Die Studie, deren Ergebnisse bald im Swiss Medical Weekly publiziert werden, zeigt gewisse Abstriche bei der Qualität, eine Verschiebung hin zu mehr administrativen Tätigkeiten und durch ökonomischen Druck verursachte Interessenkonflikte. Ein kleiner, aber doch signifikanter Anteil der Kolleginnen und Kollegen verspürt im Augenblick Druck, ökonomisch zu agieren und sich primär ökonomisch als am Patientenwohl zu orientieren. Die Teilnehmenden nehmen Veränderungen eher zum Schlechteren hin wahr. Die Frage, ob die Kernprinzipien medizinethischer Standards in der klinischen Alltagstätigkeit umgesetzt werden können, wird von der Mehrheit als «nur mit Einschränkungen umsetzbar» wahrgenommen.

Trotz solcher Konsequenzen von Anreizsystemen wie DRGs kann nicht auf sie verzichtet werden. Es sollte jedoch das Bewusstsein für die Konsequenzen von Anreizsystemen geschärft werden, die als lernende Systeme zu konzipieren sind, was bedingt, dass negative Auswirkungen so früh als möglich erkannt werden.

Ein Schlüssel, um negative Konsequenzen einer fehlgeleiteten Ökonomisierung zu verhindern, liegt nach Prof. Biller-Andorno im Model «Triple Aim», das vom Institute of Healthcare Improvement in Boston entwickelt wurde und welches die amerikanische Gesetzesentwicklung mit dem Affordable Care Act beeinflusst hat. Dieses Modell zeigt, dass die Gesundheitsversorgung immer drei Dimensionen hat und das Ziel nur erreicht ist, wenn alle drei Dimensionen gelingen: neben der (a) individuellen Patientenerfahrung in der Versorgung geht es auch darum (b) den Gesundheitszustand der Bevölkerung zu verbessern sowie (c) die Gesundheitskosten zu reduzieren. Als Schritte in diese Richtung sieht die Referentin den vermehrten aktiven Einbezug der Patienten, gerade wenn es im Sinne eines shared decision makings darum geht, maximale von optimaler Versorgung zu unterscheiden, und die Schaffung einer Kultur, die Offenheit und kritische Reflexion fördert und damit auch die Verbesserung der Vergütungs- und Anreizstrukturen ermöglicht. Weitere Schritte wären auch die Berücksichtigung der Ethikkultur in den bestehenden Zertifizierungsprozessen sowie das gezielte Auswerten und Erheben von Daten (Versorgungsforschung, Monitoring, Patientenerfahrungen).

Marc Müller/Hausärzte Schweiz fragt, ob es in der Schweiz vergleichbare Untersuchungen oder Daten aus dem Praxisalltag gibt.

Nikola Biller-Andorno verneint dies. Es ist jedoch ein Projekt, dem sie sich gerne als nächstes zuwenden möchte.

Ricardo Torriani/ZH erwähnt das Beispiel aus einem Zentrumsspital. Dieses führt Kataraktoperationen nur in Narkose durch, weil die Patienten zufriedener sind. Die Kosten sind jedoch deutlich höher.

Nach Nikola Biller-Andorno ist dies ein zentrales Dilemma und eine primäre Herausforderung, noch mehr Aufklärungsarbeit zu leisten. Das kann im individuellen Arzt-Patient-Gespräch sein, indem die Behandlungsoptionen, die Risiken wie auch die Kosten aufgezeigt werden. Zum einen gibt es vielleicht das Narkoserisiko in dem Fall, zum anderen aber auch die höheren Kosten. Es braucht in der Frage einer optimalen Versorgung und Kosten-Nutzen-Verhältnis einen anderen Diskurs als heute.

Marianna Bodenmann/ZH interessiert, ob es einen Unterschied zwischen Männern und Frauen bei den 


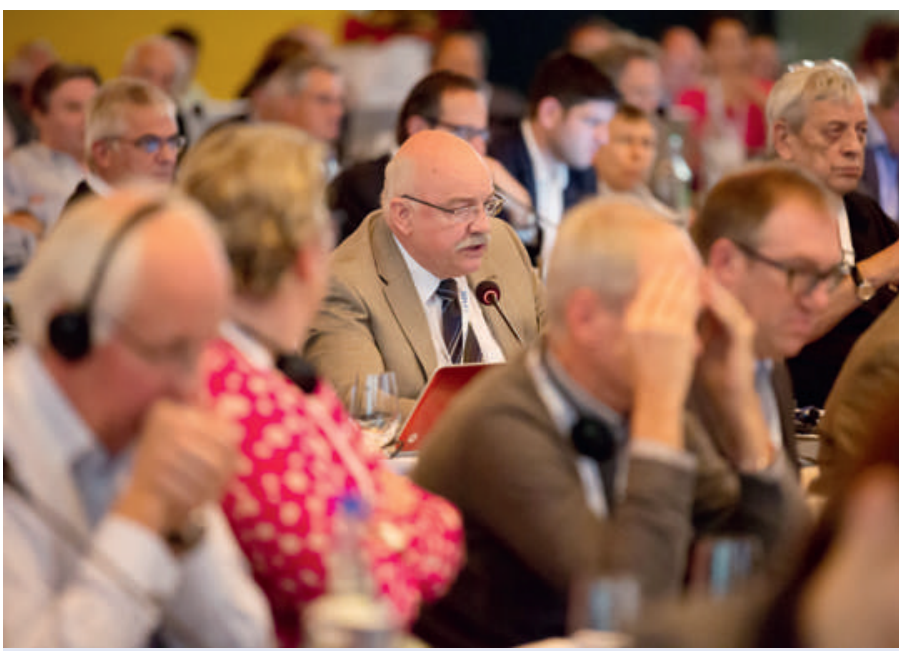

Im Dialog: die Delegierten der Ärztekammer ...

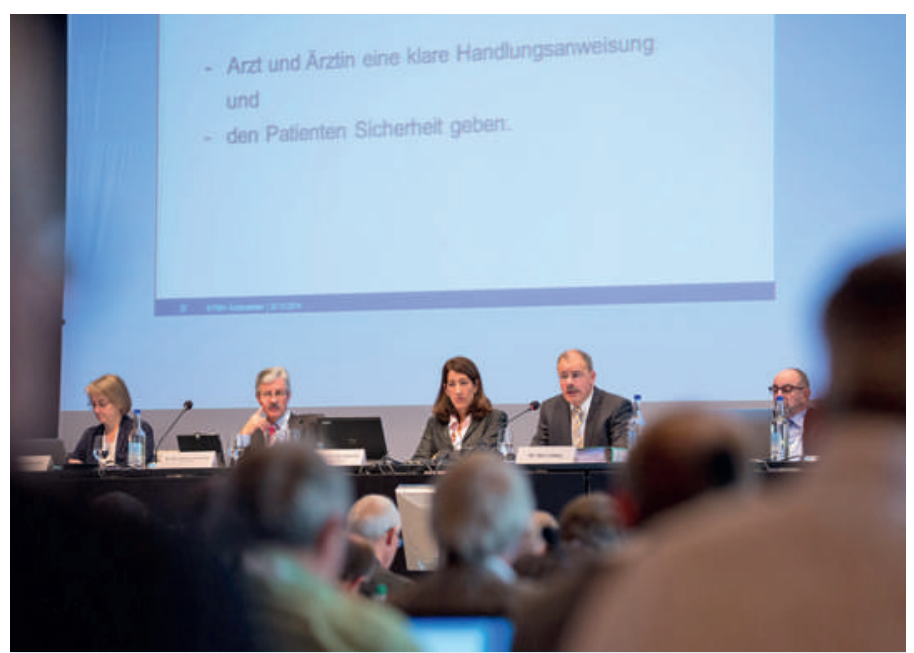

... und der Zentralvorstand der FMH.
Ärztinnen und Ärzten gibt, wie sie die Medizin erleben, und ob es einen Unterschied in der Behandlung von Patientinnen gibt? Medikamente werden ja vor allem nur an Männern getestet und bei älteren Frauen angewandt.

Nikola Biller-Andorno hält fest, dass in ihrer Studie bei den Geschlechtsunterschieden keine Ungleichheit gefunden wurde. Auch bei den Hauptdisziplinen gab es keine signifikanten Unterschiede. Wenn jedoch der Fokus explizit auf Geschlechterunterschiede gerichtet sein soll, muss die Studie für aussagekräftigere Resultate anders ausgelegt sein.

Anja Zyska/VSAO fragt, ob eine ähnliche Studie über Ökonomie und Kosten der Medizin und Qualität vor Einführung des Swiss DRG durchgeführt wurde und ob es Vergleiche gibt, in wie weit sich das verändert hat?

Nikola Biller-Andorno kennt keine Studie, die ähnliche Fragen in dieser empirischen Weise gestellt hat. Kurz vor Einführung der DRGs, sozusagen um die Stunde Null, wurde eine ähnliche Umfrage durchgeführt. Die Auswertung ist noch nicht abgeschlossen.

Brigitte Muff/AG will wissen, ob es Hinweise dafür gibt, dass die Ärzte in den Kliniken zunehmend verwaltet werden und die Mitbestimmung in der Leitung abgenommen hat und ob das Management heute anders zusammengesetzt ist als vor wenigen Jahren?

Nikola Biller-Andorno führt aus, dass einige auf die Frage: «Habe ich sozusagen einen Patienten aufgrund von Druck aus dem Management anders behandelt, als ich es hätte tun wollen» doch geantwortet haben, dass sie diesen Druck aktiv aus dem Management spüren. Bei einer weiteren Frage «Könnt ihr euch entsprechend den medizinischen Prinzipien verhalten» wurde auch eine Einschränkung wahrgenommen. Nach ihr wäre es wichtig, im eigenen Spital zu beobachten, ob die Ärzteschaft oder auch die Pflege einen Druck aus dem Management erlebt? Auf die Auswirkung einer anderen Zusammensetzung im Management wurde in der Studie nicht Wert gelegt. Die Frage der Gewichtung - Klinik versus Ökonomie - ist natürlich ein aktuelles Thema.

Jürg Schlup dankt Prof. Biller-Andorno herzlich für ihr Kommen und den ausgezeichneten spannenden Vortrag. Er verweist auf den McKinsey Bericht «Management in Healthcare von 2010», wo es um die bestgeführten Spitäler ging. Eine der Empfehlungen daraus, «Encourage more movement of clinical staff into management» zeigt einen neuen Trend im angelsächsischen Raum. Es braucht mehr Ärzte und Pflegende in der Spitalleitung.

Pierre-François Cuénoud/ZV FMH informiert bei dieser Gelegenheit, dass das gfs.bern im Auftrag der FMH jährlich eine repräsentative Befragung der Ärzteschaft zu den Auswirkungen der neuen Spitalfinanzierung im allgemeinen sowie zur Einführung von SwissDRG im speziellen durchführt. Untersucht wurde z. B. die Entwicklung des administrativen Aufwands, der Behandlungsfreiheit usw. www.fmh.ch $\rightarrow$ Stationäre Tarife $\rightarrow$ Begleitforschung $\rightarrow$ Entwicklung Rahmenbedingungen Ärzteschaft.

Der Präsident dankt an dieser Stelle allen Mitarbeiterinnen und Mitarbeitern der FMH für ihre Arbeit an der ÄK. Dank ihnen ist es möglich, diese Veranstaltung zielgerichtet und effizient durchzuführen.

\section{Budgets 2015 von SIWF, GPK und FMH}

\subsection{Budget 2015 SIWF}

Werner Bauer/Präsident SIWF nutzt die Gelegenheit, einige Bemerkungen zum Budget SIWF und Informationen zu aktuellen Projekten und Schwerpunktthemen zu geben. Das Budget SIWF schliesst dieses Jahr, wie bereits früher angekündigt, mit einem Defizit ab. In den letzten Jahren waren die Einnahmen jeweils durch die ansteigende Zahl der Diplomierungen hoch, was sich nun geändert hat. Ziel des SIWF ist es, sein Vermögen für sinnvolle Projekte in der Weiterbildung und in der Unterstützung der Weiterbildenden zu nutzen und kein Geld anzuhäufen. Mit dem 
Award konnten Weiterbildende für ihr Engagement in der Weiterbildung ausgezeichnet werden. Mit dem Projekt Förderung der Weiterbildung wurden von den 62 eingereichten Projekten schlussendlich vier Projekte berücksichtigt (PathoBasic, Ausbildung in minimalinvasiver Chirurgie, RapRad - eine Radiologie-Lernplattform, Beatmungssimulation mit der TestChest $^{\mathrm{TM}}$ ). Dieses Jahr wurde erstmals und mit grossem Erfolg das Medical Education Symposium durchgeführt. Schlussendlich ist das SIWF zusammen mit Bund und GDK in einer Arbeitsgruppe vertreten, die sich mit der Steuerung der Facharztweiterbildung befasst.

Christoph Hänggeli/SIWF informiert über den Stand der aktuellen IT-Projekte. Beim e-Logbuch sind bereits 30 eidgenössische Facharzttitel und 11 Schwerpunkte eingepflegt. Die letzten Module sind in Entwicklung und am 1. Juli 2015 sollten alle Fachgebiete aufgeschaltet sein. Dieses Jahr soll das Cockpit für die Titelkommission und nächstes Jahr das Cockpit für die Weiterbildner realisiert werden. Weitere budgetrelevante Projekte sind die Fortbildungsplattform und die webbasierte Datenerfassung bei den Weiterbildungsstätten. Bei der Revision des Medizinalberufegesetzes konnten zwei wichtige Anliegen eingebracht werden; zum einen das vollständige Ärzteregister und zum anderen der Antrag, dass eine Landessprache wenigstens auf Niveau B2 beherrscht wird. Bei letzterem Anliegen ist der Entscheid des Ständerats noch offen

Wie schon erwähnt, weist das Budget SIWF 2015 einen Verlust von CHF 921000 auf. Bei einem gleichbleibenden Ertrag ist der Mehraufwand deutlich gestiegen. Gründe sind u.a. der wesentlich höhere Mehraufwand bei den IT-Projekten e-Logbuch, Fortbildungsplattform und bei den Personalkosten. Das SIWF verfügt jedoch über Reserven von CHF 6 Mio. welche erlauben, den budgetierten Verlust auszugleichen und die geplanten Projekte zu realisieren.

Nach Aufforderung des Präsidenten gibt es keine Fragen oder Wortmeldungen zum Budget SIWF.

\subsection{Budget $2015 \mathrm{FMH}$}

Emanuel Waeber/FMH weist darauf hin, dass es trotz tiefgreifenden Sparmassnahmen nicht gelungen ist, der ÄK ein ausgeglichenes Budget zu präsentieren. Das Budget FMH weist für 2015 ein Defizit von CHF 1004431 aus beziehungsweise CHF 691931 bei Zustimmung zum Sonderbeitrag zugunsten der Gesamtrevision TARMED. Zusammen mit jenem des SIWF ist nächstes Jahr von einem Verlust von CHF 1926000 auszugehen. Das vorliegende Budget wurde erstmals unter Berücksichtigung der neu eingeführten mittel- und langfristigen Finanzplanung 2016-2018 erstellt. Die Finanzplanung soll gewährleisten, dass einerseits mittel- und langfristige Projekte auf der Zeitachse und aufwandseitig einer optimaleren Planung unterliegen und es der FMH anderseits erlauben, bei der Umsetzung die entsprechenden
Prioritäten festzulegen. Die Einnahmen stiegen in den letzten fünf Jahren aufgrund der Zunahme bei den ordentlichen Mitgliederbeiträgen um 19,9\%. Die Ausgaben nahmen seit 2009 um 36,7\% zu. Die Resultate aus der neuen Finanzplanung haben den ZV bewogen, als erste Massnahme ab 2016 eine Ausgabenbremse einzuführen. Mit diesem Instrument soll erreicht werden, die Ausgaben so zu limitieren, dass diese die Einnahmen nicht mehr übersteigen. Ebenso sind die Aufgaben zu überprüfen. Die Bildung neuer Kommissionen mit direkter Kostenfolge auf Entschädigungen wird nur bei Vorliegen eines durch den ZV genehmigten Projektantrags bewilligt.

\subsection{Budget 2015 GPK}

Emanuel Waeber/FMH präsentiert das Budget der GPK, welches bei einem Gesamtaufwand in der Höhe von CHF 96000 im Vergleich zu 2014 um CHF 5000 tiefer sein wird.

Jürg Schlup/Präsident FMH gibt den Anwesenden die Möglichkeit für Fragen und Bemerkungen. Da dies nicht der Fall ist, werden vor der Schlussabstimmung die Anträge für die Sonderbeiträge NAKO und IPI behandelt.

\subsection{Sonderbeiträge FMH}

\subsubsection{Sonderbeitrag NAKO (NewIndex)}

Die Datensammlung der praktizierenden Ärzte trägt wesentlich zur guten Datenlage der Ärzteschaft bei. Die Auswertungen auf kantonaler, regionaler und nationaler Ebene ermöglichen es, auf Augenhöhe mit den anderen Stakeholdern im Gesundheitswesen zu verhandeln und damit auch Erfolge für die Ärzteschaft zu erzielen. Ernst Gähler/ZV FMH weist darauf hin, dass mit der subsidiären Kompetenz des Bundesrates für den Eingriff in die Tarifstruktur TARMED und mit der geplanten Nachfolgelösung des Bundes zum Zulassungsstopp in die kantonalen Taxpunktwerte es noch wichtiger geworden ist, dass die Ärzteschaft über eine umfassende, qualitativ gute und repräsentative Datensammlung verfügt. Ohne diese Daten können die Kostenmodelle nicht aktualisiert werden. Die FMH hat einen eigenen Tarifbrowser entwickelt. Der Sonderbeitrag dient vor allem der Basisfinanzierung und der Sicherstellung der Betriebsinfrastruktur und ermöglicht der Ärzteschaft einen laufenden Zugang zum NAKO-System.

Franziska Zogg/ZG möchte an der nächsten ÄK, wenn es wiederum um den Sonderbeitrag NAKO geht, die entsprechenden Finanzflüsse sehen.

Ernst Gähler/ZV FMH versichert, dass der Sonderbeitrag nicht in Trust Center fliesst, sondern einzig und allein der Aufbereitung der Daten sowie zur Basisfinanzierung der Arbeiten bei NewIndex dient.

\subsubsection{Sonderbeitrag IPI}

Emanuel Waeber/FMH erinnert daran, dass die ÄK heute den Leistungsvertrag IPI um ein weiteres Jahr 
verlängert hat. Das IPI soll die konkrete Praxisinformatik auch für das Jahr 2015 weiterführen. Zur Finanzierung soll ein Sonderbeitrag von CHF 30 pro FMH-Mitglied der Kategorien 1 bis 6 erhoben werden.

Da es keine Fragen zu den Sonderbeiträgen gibt, erteilt Jürg Schlup dem Präsidenten der GPK das Wort.

Adrian Sury/Präsident GPK empfiehlt der ÄK die Annahme des Budgets 2015. Die GPK hat sich intensiv mit der Budgetlage auseinandergesetzt und entsprechende Massnahmen für 2016 vorgeschlagen, die im Folgetraktandum diskutiert werden. Seiner Meinung nach macht es keinen Sinn, das Budget 2015 im jetzigen Zeitpunkt abzulehnen.

Da es keine weiteren Wortmeldungen gibt, wird wie folgt über die Budgets abgestimmt.

- Budget 2015 SIWF

Das Budget wird mit 134 Ja und 12 Enthaltungen ohne Gegenstimme genehmigt.

- Budget 2015 FMH

Das Budget wird mit 93 Ja zu 11 Nein und 35 Enthaltungen genehmigt.

- Einführung Ausgabenbremse ab Budget 2016: Die Gesamthöhe der im Budget aufgeführten Ausgaben der FMH (ohne SIWF) darf die jeweiligen Einnahmen (Mitgliederbeiträge und Dienstleistungserträge) des letzten genehmigten Jahresabschlusses nicht übersteigen.

Die Einführung einer Ausgabenbremse ab Budget 2016 wird mit $128 \mathrm{Ja}$ zu 11 Nein und 5 Enthaltungen angenommen.

- Bildung neuer Kommissionen mit direkter Kostenfolge auf Entschädigungen wird nur bei Vorliegen eines durch den $Z V$ genehmigten Projektantrags bewilligt.
Der Antrag wird mit 135 Ja zu 2 Nein und 11 Enthaltungen angenommen.

\section{- Sonderbeitrag NAKO}

Der Antrag um Erhebung eines zweckgebundenen Sonderbeitrags von CHF 40 zugunsten der Nationalen Konsolidierungsstelle NAKO bei den Mitgliederkategorien 1 und 2 wird mit $106 \mathrm{Ja} z u$ 1 Nein und 37 Enthaltungen genehmigt.

Der Intervention von Thomas Heuberger/BE, wonach es bei der Budgetberatung keine Möglichkeit für eine Diskussion gegeben hat, hält Konrad Schiess/SG entgegen, dass jeder beim Aufruf des Präsidenten hätte Fragen stellen oder intervenieren können. Er stellt den Ordnungsantrag, dass die Diskussion betreffend Budget als beendet betrachtet gilt.

\section{- Ordnungsantrag Konrad Schiess/SG}

Die Diskussion zum Budget 2015 hat stattgefunden und wird als beendet betrachtet. Die bisherigen Abstimmungen zum Budget sind erledigt.

Beschluss:

Dem Antrag wird mit 100 Ja zu 9 Nein und 13 Enthaltungen zugestimmt.

Bevor über den Sonderbeitrag IPI abgestimmt wird, gibt es weitere Wortmeldungen.

Pierre-Alain Schneider/GE macht den Vorschlag, dass bei Annahme des Sonderbeitrages IPI entschieden wird, dass die durch diesen Sonderbeitrag finanzierten Projekte ins Departement eHealth integriert werden.

Marc Müller/Hausärzte Schweiz stellt eine Verständnisfrage. Der Sonderbeitrag wird ja von der FMH eingefordert. Der Leistungsvertrag besteht zwischen der FMH und dem IPI und die FMH bezahlt das IPI gemäss Abmachung für die entsprechenden Aufträge und Projekte. Für ihn ist unklar, was man nachträglich noch regeln möchte.
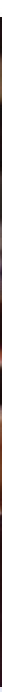

Jede Stimmte zählt: Die 200 stimmberechtigten Delegierten vertreten ihre Basis- und Fachorganisationen an der Ärztekammer. 
Hanspeter Kuhn/FMH versteht den Antrag so, dass bei Annahme dieses Vorschlags das Departement eHealth entscheiden müsste, welche Projekte jetzt unterstützt werden. Das wäre dann nach seinem Verständnis ein Rückkommensantrag auf die bereits stattgefundene Abstimmung über die Verlängerung des Leistungsvertrages, wo auch die Projekte genehmigt wurden. Der Antrag müsste präzisiert werden.

Pierre-Alain Schneider/GE hält an seinem Vorschlag fest. Es ist für ihn wichtig, dass das IPI ins Departement eHealth integriert wird und dass der Sonderbeitrag auch dafür genutzt wird. Da er die Details des Leistungsvertrages nicht kennt, ist er der Meinung, dass es sich bei den finanzierten Projekten um solche handelt, die mit dem Departement eHealth koordiniert werden müssen.

Anne-Geneviève Bütikofer/GS FMH erklärt, dass die von der FMH finanzierten Projekte dem IPI und nicht der FMH gehören. Die FMH finanziert eine Leistung, die zu erbringen ist.

Pierre-Alain Schneider/GE erscheint die Situation nach wie vor schwierig und unklar. Für ihn ist die Klärung dieser Frage wie die Integration des IPI ins Departement eHealth äusserst wichtig. Er zieht seinen Antrag zurück.

Stefan Greuter/VSAO unterstützt die Projekte des IPI. Er sieht jedoch die Auslagerung der Kernaufgaben längerfristig als Risiko. Der Aufbau einer Parallelstruktur ist ineffizient und kostenintensiv. Er hat heute zur Kenntnis genommen, dass in der Projektliste des IPI ebenfalls die Integration des IPI in die eHealthGruppe der FMH vorgesehen ist. Hier sieht er auch ein mögliches Sparpotential. Er unterstützt den Antrag Schneider und ergänzt diesen mit dem Zusatz, dass das Projekt «Integration vom IPI in die FMH» ins Departement eHealth 2015 prioritär behandelt wird und die Integration im 2016 vollzogen ist.

Jürg Schlup/Präsident FMH erinnert daran, dass es hier um die Budgetdebatte geht. Der Antrag Greuter könne nicht an dieser Stelle behandelt werden, bittet jedoch den Rechtsdienst um Klärung der Situation. Er führt weiter an, dass er den Vorschlag gerne zuhanden Weiterbearbeitung im ZV entgegennehme.

Hanspeter Kuhn/FMH hält fest, dass die Abstimmung über den Leistungsvertrag IPI bereits erfolgt ist. Beim jetzigen Antrag geht es um die Verpflichtung der FMH, dem IPI einen Sonderbeitrag zu sprechen. Würde dieser Antrag abgelehnt, stellt sich die Frage nach einem Rückkommensantrag. Die ÄK kann immer wieder auf bereits gefällte Beschlüsse zurückkommen. Es handelt sich hier um zwei Anliegen, die nicht vermischt werden können.

Jürg Schlup/Präsident FMH fragt Stefan Greuter, ob er einverstanden wäre, wenn der ZV vorerst den Antrag diskutiert und an der nächsten ÄK entsprechende Anträge vorlegt.

Stefan Greuter/VSAO erkundigt sich, ob an einen Sonderbeitrag auch Bedingungen geknüpft werden können. Wenn ja, möchte er den Antrag wie folgt for- mulieren: Wird der Sonderbeitrag IPI angenommen, so ist dies an zwei Bedingungen zu knüpfen. Erstens ist im Jahr 2015 das Projekt «Integration IPI in das Departement eHealth der FMH» mit dem Ziel einer Integration im Jahre 2016 prioritär zu behandeln. Zweitens sind die Fortschritte des Projektes an der nächsten Ärztekammer in einem halben Jahr zu präsentieren.

Da Pierre-Alain Schneider/GE seinen Antrag zurückzieht, wird über den Antrag VSAO und den Antrag ZV betreffend Sonderbeitrag IPI abgestimmt.

\section{Antrag Greuter/VSAO:}

Wird der Sonderbeitrag IPI angenommen, so ist dies an zwei Bedingungen zu knüpfen. Erstens ist im Jahr 2015 das Projekt «Integration IPI in das Departement eHealth der FMH» mit dem Ziel einer Integration im Jahre 2016 prioritär zu behandeln. Zweitens sind die Fortschritte des Projektes an der nächsten Ärztekammer in einem halben Jahr zu präsentieren.

\section{Beschluss:}

Der Antrag wird mit $100 \mathrm{Ja} \mathrm{zu} 44$ Nein angenommen.

\section{Antrag Sonderbeitrag IPI}

Die FMH bestätigt die Weiterführung der finanziellen Unterstützung zugunsten des IPI für das Gesamtmandat in den Bereichen der elektronischen Krankengeschichte (eKG) und die konkrete Praxisinformatik auch für 2015 mit einem Sonderbeitrag in der Höhe von CHF 30 pro FMH-Mitglied der Kategorien 1 bis 6 zu unterstützen.

\section{Beschluss:}

Der Sonderbeitrag IPI wird mit 91 Ja zu 46 Nein und 7 Enthaltungen genehmigt.

\subsection{Massnahmen zur Budgetstabilisierung}

Jürg Schlup/Präsident FMH informiert, dass zu diesem Traktandum vier weitere Anträge eingereicht wurden. Er bittet die Antragsteller, diese zu präsentieren. Die jeweiligen Abstimmungen werden am Schluss durchgeführt.

\subsubsection{Massnahmen zur Budgetstabilisierung Antrag GPK}

Adrian Sury/Präsident GPK zeigt sich besorgt über die düsteren finanziellen Aussichten der FMH. Heute sind bereits 95\% der Einnahmen für gebundene Ausgaben reserviert. Dadurch besteht kaum Handlungsfreiraum für neue dringende Aufgaben. Trotz grosser Anstrengungen im GS, das Defizit des Budgets $2015 \mathrm{zu}$ reduzieren, besteht eine grosse Diskrepanz zwischen Einnahmen und Ausnahmen. Die Aufgabenüberprüfung durch den ZV brachte keinen Erfolg. Die FMH soll und muss sich auch künftig als bedeutende und einflussreiche Organisation positionieren. Dafür braucht es Geld, das aufgrund der aktuellen finanziellen Situation fehlt. Um die Organisation wirtschaftlich mittel- und langfristig wieder auf ein solides 


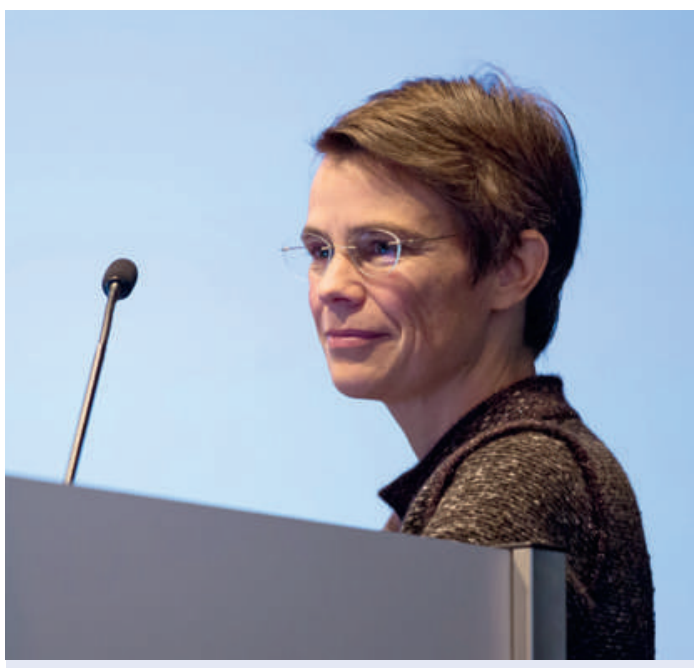

Gastreferentin war Prof. Nikola Biller-Andorno, Institutsdirektorin und Professorin für Biomedizinische Ethik der Universität Zürich.

Fundament zu stellen, braucht es dringliche Massnahmen. Die GPK ist der Ansicht, dass sowohl DV wie Ärzteschaft schwerlich in der Lage sind, entsprechende Vorgaben zu machen. Ziel ist, die Ausgaben und Einnahmen auf Dauer im Gleichgewicht zu halten wie auch einen operativen Freiraum von 20\% der Ausgaben für priorisierte Projekte zu schaffen. Die heute bereits beschlossenen Massnahmen alleine reichen nicht aus. Die GPK schlägt deshalb als weitere Massnahme die Bildung einer Task Force vor. Diese soll die Aufgaben der FMH überprüfen sowie priorisieren und die Ausgaben der FMH in einem von der ÄK zu bestimmenden Mass reduzieren. Aus Sicht der GPK sollten in der Task Force Mitglieder mit politischer und ökonomischer Kompetenz Einsitz nehmen. Sie schlägt folgende Zusammensetzung vor: Jürg Schlup/Präsident FMH, Peter Wiedersheim/Präsident DV, Adrian Sury/Präsident GPK, Thomas Kehl/Mitglied GPK, Anne-Geneviève Bütikofer/GS FMH und eine externe Moderation. Thomas Kehl gilt als Vertreter der fmCh und verfügt als CEO von zwei Kliniken über grosse ökonomische Kompetenz. Die GPK ist der Ansicht, dass zuerst die Aufgaben priorisiert und in einem zweiten Schritt Vorschläge für weitere Einnahmen geprüft werden müssen. Die Erhöhung der Mitgliederbeiträge kann im Anschluss daran ebenfalls in Erwägung gezogen werden.

Die GPK stellt aus den vorherigen Folgerungen folgenden Antrag:

\section{Antrag GPK:}

Die ÄK beauftragt den ZV, zur Vergrösserung des operativen Freiraums

1. Vorbereitet durch eine Task Force, die Aufgaben der FMH zu prüfen und zu priorisieren

2. Die Ausgaben der FMH in einem von der ÄK zu bestimmenden Mass zu reduzieren. Erst in einem weiteren Schritt müssen, wenn nötig, zusätzliche Einnah- men generiert werden und allenfalls eine Erhöhung des Mitgliederbeitrags in Erwägung gezogen werden.

Jean-Pierre Vuillemin/VD findet die Aussage, dass DV und $\mathrm{AKK}$ nicht in der Lage seien, eine Beurteilung abzugeben, widersprüchlich. Die GPK verlangt ja von der ÄK, Entscheidungen zu treffen. Auf einer Seite fühlt er sich nach den Worten von Adrian Sury inkompetent, auf der anderen Seite jedoch soll er dann kompetent als Mitglied der ÄK Entscheide fällen. Er schlägt vor, zuerst die Ausgaben zu senken und erst in einem nächsten Schritt die Erhöhung der Mitgliederbeiträge zu prüfen.

Adrian Sury/Präsident GPK zweifelt nicht an der Kompetenz der ÄK-Delegierten. Tatsache ist, dass die ÄK-Delegierten verschiedene, teilweise divergierende Interessen zu vertreten haben. Anderseits liegt die Finanzkompetenz bei der ÄK. Für ihn ist dies keine Kontradiktion.

Beat Gafner/BE findet die Bildung der Task Force eine ausgezeichnete Idee und will wissen, ob die von der GPK präsentierte Zusammensetzung abschliessend oder als Vorschlag zu verstehen ist.

Adrian Sury/Präsident GPK würde eine solche $\mathrm{Zu}$ sammensetzung begrüssen. Es gibt heute zu der $\mathrm{Zu}$ sammensetzung noch einen weiteren Antrag, doch er wünscht, dass über den Vorschlag der GPK abgestimmt wird. Natürlich kann die Task Force noch mit weiteren Personen ergänzt werden.

Marc Müller/Hausärzte Schweiz hat nach den vorgängigen Aussagen den Eindruck, dass die FMH ein Globalbudget einführen will. Nach ihm sollte die FMH sich zuerst über die Kernaufgaben und auf jene Aufgaben beschränken, die zwingend finanziert werden müssen. Er weist darauf hin, dass pharmaSuisse die Mitgliederbeiträge nach dem Umsatz festlegt, was ihr die geforderte Durchsetzungskraft bei der Erreichung ihrer Projekte ermöglicht.

Adrian Sury/Präsident GPK bekräftigt nochmals, dass die Task Force die Aufgabenüberprüfung vornehmen soll. Anschliessend ist es an der ÄK, die nötigen Entscheide zu fällen. Es geht hier nicht um die Einführung eines Globalbudgets, sondern darum, das vorhandene Geld intelligent einzusetzen. Heute wurde die Integration des IPI in die FMH per 2016 entschieden. Die GPK hat diesen Vorschlag, auch im Hinblick auf Einsparung von Ressourcen, bereits früher eingebracht.

Charles Favrod-Coune/VD unterstützt den Antrag der GPK, schlägt jedoch vor, die Task Force mit weiteren Mitgliedern aus der Basis zu verstärken.

\subsubsection{Antrag zur Unterstützung des Antrags der GPK (fmCh)}

Urban Laffer/Präsident fmCh unterstützt den Antrag der GPK, dringende Massnahmen zur Stabilisierung des FMH-Budgets zu ergreifen und empfiehlt der ÄK, diesem Antrag vorbehaltlos zuzustimmen. 
Antrag fmCh:

Die fmCh unterstützt den Antrag der GPK, dringende Massnahmen zur Stabilisierung des FMH-Budgets zu ergreifen und empfiehlt der ÄK, diesem Antrag vorbehaltlos zuzustimmen.

6.5.3 Abbau des strukturellen Defizits der FMH (Antrag J. Nadig)

Jürg Nadig/SFSM und weitere mitunterzeichnende Gesellschaften wollen mit ihrem Antrag die Wiederherstellung des finanziellen Gleichgewichtes der FMH, damit die Ärzteschaft die in der Strategie 2020plus festgelegten Ziele erreichen kann. Dieses Gleichgewicht kann mittelfristig durch Abbau von Nebenaufgaben und durch neue Finanzierungsmodelle und -quellen wiederhergestellt werden. Die ÄK hat bereits vor zwei Jahren entschieden, die FMH zu professionalisieren. Dieser Weg sollte nun in allen Departementen weitergegangen werden. Eine Professionalisierung ist mit Kosten verbunden und bedingt neue Finanzierungquellen. In diesem Sinne beantragen sie sowohl neue Mittel wie auch die Kontrolle der Ausgaben.

\section{Antrag Nadig:}

Die Ärztekammer bestätigt das Strategieziel «Gesundheitspolitik immer mit der FMH» und «die FMH geht Entwicklungen im Gesundheitswesen proaktiv an». Um dieses zu erreichen, beauftragt sie den ZV zusammen mit der DV bis zur nächsten Ärztekammersitzung einerseits Vorschläge auszuarbeiten, welche Nebenaufgaben der FMH in welchen Zeitrahmen abzubauen sind und andererseits neue Finanzierungsmodelle auszuarbeiten, damit das strukturelle Defizit ab 2016 nachhaltig abgebaut werden kann und die FMH weiterhin die Anliegen der Ärzteschaft in der Gesundheitspolitik vertreten und als kompetenter Partner das Gesundheitswesen mitgestalten kann.

\subsubsection{Massnahmen zur Budgetstabilisierung} Antrag Christoph Ramstein, VEDAG

Christoph Ramstein/VEDAG erläutert den Antrag, welcher sich einzig auf die Zusammensetzung der Task Force bezieht. Der VEDAG wünscht eine andere Zusammensetzung als die GPK. Er ist der Meinung, dass in der Task Force neben dem GS, der GPK auch Vertreter der fmCh, des VSAO und der KKA braucht. Bei den letztgenannten sollte jemand aus der Romandie berücksichtigt werden.

\section{Antrag:}

Der VEDAG beantragt für die von der GPK vorgeschlagene Task Force die folgende personelle Zusammensetzung:

- 1 externe Fachperson (Ökonom/Rechnungsprüfer)*

- 1 Vertreter GS

- GPK-Präsident (Vorsitz)

- 1 Vertreter $\mathrm{fmCh}^{* *}$

- 1 Vertreter VSAO ${ }^{* *}$

- 1 Vertreter KKA ** (** aus Romandie)
* Vorschlag: aus BSG Unternehmungsberatung AG St. Gallen (sind mit der Materie weitgehend vertraut. Sie waren bei der Beratung der Führungsmodelle miteingebunden.

${ }^{* *}$ mindestens 1 Person aus diesen Vertretungen muss aus der Romandie kommen

Der FMH-Präsident kann an den Sitzungen mit beratender Stimme teilnehmen.

Die Task Force unterbreitet via GPK der Ärztekammer ihre Vorschläge und Anträge zu Handen der Ärztekammersitzung vom 29. Oktober 2015.

Nach den verschiedenen Wortmeldungen betreffend Zusammensetzung der Task Force fasst Jürg Schlup/ Präsident FMH die präsentierten Anträge zusammen. Es geht nun darum, Entscheide zu den Fragen, ob und welche Task Force, Zeitpunkt der Präsentationen der Vorschläge an der ÄK sowie über Ziele zu fällen.

Die vier Antragsteller, GPK, fmCh, J. Nadig und VEDAG sind mit diesem Vorgehen einverstanden.

Pius Bucher/TG beantragt, die Ziele als zweiten Punkt zu behandeln.

Jürg Schlup/Präsident FMH ist mit dem Vorgehen einverstanden und erwähnt, dass der Vertreter der VEDAG bereits ausgeführt hat, dass ihre Ziele mit jenen der GPK ähnlich sind. Die fmCh unterstützt ebenfalls die Ziele der GPK. Die Diskussion betreffend Ziele GPK versus Ziele SFSM ist eröffnet.

Christian Bernath/FMPP findet die Fragestellung, welche Aufgaben die FMH übernehmen soll, zu wenig präzis. Die Grundsatzfrage ist vielmehr, welche Aufgaben die FMH zusätzlich übernehmen soll, um eine stärkere Position im Gesundheitswesen einzunehmen. Es geht nicht allein um den Abbau von Nebenaufgaben.

Jürg Schlup/Präsident FMH weist darauf hin, dass diese Fragestellung im Antrag der GPK beinhaltet ist.

Hans-Anton Vogel/VEDAG meint, dass die finanziellen Mittel mit der Zunahme der Aufgaben heute fehlen. Vor 20 Jahren war die FMH eine reiche Organisation, die mit dem Kauf von Immobilien und mit Inseraten Geld generiert hat. Es gilt zu überlegen, ob die Finanzen der FMH nur noch aus den eigenen Beiträgen zu erwirtschaften sind, oder ob sich auch neue Finanzquellen eröffnen sollen.

Stefan Greuter/VSAO findet die Zielsetzung gut. Was ihm jedoch fehlt, ist ein Blick in die Zukunft. Er ist der Meinung, einen Teil des Budgets für Zukunftsprojekte, wie z.B. Sicherstellung des Ärztenachwuchs, bereitzustellen.

Jürg Pfisterer/SGIM erinnert daran, dass die FMH ein Leitbild mit Zielen verabschiedet hat. Er regt an, das Leitbild der FMH für die weiteren Diskussionen betreffend Aufgaben FMH einzubeziehen.

Jürg Schlup/Präsident FMH entgegnet, dass die Aufgabenliste im Traktandum 9 viel detaillierter und basisnaher ist, als im Leitbild. Das Leitbild gibt eine 
Richtung an und dieser Richtung sollte gefolgt werden. Er schlägt vor, dass die Task Force bei ihrer Aufgabe die im Antrag der GPK formulierten Zielen unter Berücksichtigung des Leitbilds der FMH verfolgt. Es wird wie folgt über die Anträge abgestimmt:

- Antrag Genehmigung Ziele Task Force unter Einbezug des Leitbilds FMH:

Beschluss:

Der Antrag wird mit 132 Ja, ohne Gegenstimmen und 2 Enthaltungen gutgeheissen.

- Antrag Einsetzung einer Task Force ja oder nein? Beschluss:

Der Antrag wird mit 130 Ja zu 2 Nein und 5 Enthaltungen gutgeheissen.

Vor der Abstimmung zur Zusammensetzung der Task Force gibt es weitere Wortmeldungen.

Thomas Heuberger/BE schlägt vor, dass nicht über einzelne Personen oder Fachgesellschaften, sondern über das Prinzip diskutiert wird. Der Vorschlag der VEDAG erscheint ihm sinnvoll. Die Arbeit muss von der GPK geleitet und von der Basis mitgetragen werden.

Christine Romann/ZV regt an, sich zu überlegen, welche Personen am Geeignetsten für den Einsatz in der Task Force wären. Wenn hier das Delegationsprinzip eingeführt wird, gibt es wiederum Delegierte aus verschiedenen Interessengebieten. Sinnvoller wäre, fünf Personen zu wählen, denen zugetraut wird, dass sie sich nicht von Partikularinteressen leiten lassen.

Marc Müller/Hausärzte Schweiz unterstützt das Votum von Christine Romann. Entweder wird ein Expertengremium gewählt, das volles Vertrauen geniesst oder es wird auf die gewohnte Zusammensetzung zurückgegriffen, wo jeder Dachverband eine Vertretung hat. Er stellt den Antrag für ein Expertengremium.

Jürg Schlup/Präsident FMH hält fest, dass bei der Zusammensetzung Konsens besteht, nämlich 1 Ökonom, Anne-Geneviève Bütikofer/GS FMH, Adrian

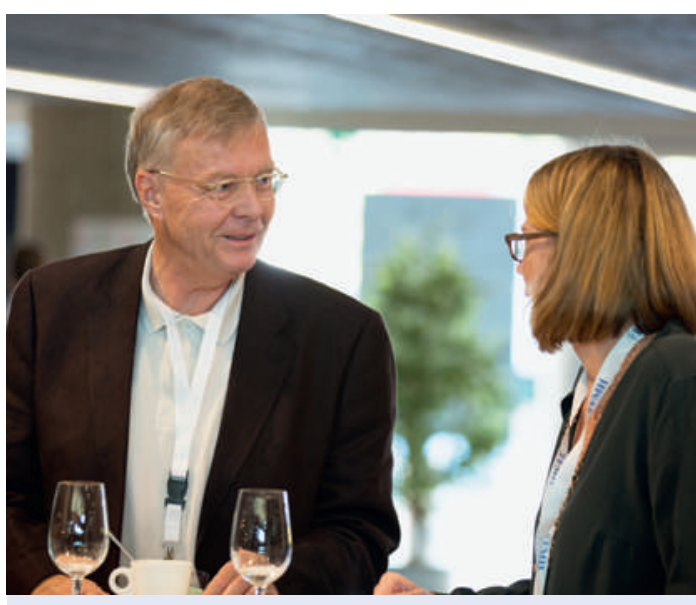

Pausen für Gespräche und Meinungsaustausch nutzen: Aus der ganzen Schweiz kommen die Teilnehmenden der Ärztekammer in Biel zusammen.
Sury/Präsident GPK, Adrian Kehl/Vertretung fmCh, eine Vertretung VSAO und eine Vertretung aus der Romandie. Es gilt nun über die Zusammensetzung der Task Force als reine Expertengruppe (Antrag Müller) versus Delegationsmodell (Anträge VEDAG und GPK) abzustimmen. Im Anschluss daran ist über die personelle Zusammensetzung wie über den Zeitpunkt der Präsentationen zu beschliessen.

- Zusammensetzung Task Force

Antrag Marc Müller: Die Task Force ist als Expertengremium mit fünf Vertretern FMH und einem Ökonom zusammengesetzt.

Antrag GPK/VEDAG: Delegationsmodell. Die EXperten sind aus der DV zu bestimmen.

Beschluss:

Für den Antrag Müller stimmen 74 Delegierte, für den Antrag GPK/VEDAG 47 Delegierte, Enthaltungen 5. Somit ist der Antrag Müller mit 74 gegen 47 Stimmen angenommen.

- Bestimmung Wahlgremium: Wahl der Experten durch DV auf Antrag ZV

Beschluss:

Der Antrag wird mit 113 Ja zu 5 Enthaltungen angenommen.

- Präsentation der Vorschläge an der ÄK Mai oder Oktober 2015:

Beschluss:

Für die Präsentation der Vorschläge an der ÄK Mai 2015 stimmen 109 Delegierte, für die Präsentation an der ÄK Oktober 2015 stimmen 11 Delegierte. Die Task Force präsentiert an der ÄK vom 7. Mai 2015 die Vorschläge.

6.5.5 Massnahmen zur Budgetstabilisierung: Antrag Hans-Anton Vogel, VEDAG

Hans-Anton Vogel/VEDAG beantragt, dass die FMH weiterhin eine schlagkräftige Organisation bleiben muss, um auch künftig bei den gesundheitspolitischen Dossiers mitzubestimmen. Um diese Aufgaben nachhaltig zu erfüllen, braucht es Mittel. Eine alleinige Reduktion der Kosten genügt nicht, vielmehr müssen bereits kurzfristig mehr finanzielle Mittel zur Verfügung stehen. Der VEDAG beantragt zuhanden des Budget 2015 einen Sonderbeitrag von CHF 100.

Schröpfer Daniel/VSAO macht beliebt, dass zuerst die Aufgaben überprüft werden und anschliessend der Entscheid über Sonderbeiträge gefällt wird. Sollte diesem Sonderbeitrag heute jedoch zugestimmt werden, stellt er den Antrag, dass man diesen nicht mehr als Sonderbeitrag ausweist, sondern eine normale Mitgliederbeitragserhöhung vornimmt.

Philippe Vuillemin/VD ist gegen einen weiteren Sonderbeitrag. Die Task Force soll ihre Aufgabe an die Hand nehmen und, wenn nötig, entsprechende Anträge für Beitragserhöhungen der ÄK unterbreiten.

Vincenzo Liguori/TI ist ebenfalls gegen die Erhebung des Sonderbeitrags. Es schlägt vor, vorerst die 
Resultate der Task Force abzuwarten, bevor Beitragserhöhungen ins Auge gefasst werden.

Es wird über die Anträge des VSAO und des VEDAG abgestimmt:

Antrag VSAO:

Sollte der Antrag VEDAG angenommen werden, wünscht der VSAO, dass dies eine Grundbeitragserhöhung und kein Sonderbeitrag ist.

Beschluss:

Der Antrag wird mit 53 Ja zu 51 Nein und 9 Enthaltungen angenommen.

Antrag VEDAG:

Der VEDAG beantragt eine umgehende und nachhaltige Verbesserung der finanziellen Lage der FMH, indem die von der GPK vorgeschlagenen Sparmassnahmen umgesetzt werden, aber schon jetzt mit einem Sonderbeitrag von CHF 100 für 2015 die Finanzen der FMH optimiert werden, bis der GPK-Bericht vorliegt.

Beschluss:

Der Antrag wird mit 31 Ja zu 78 Nein und 5 Enthaltungen abgelehnt.

\section{Bestätigungs- und Verlängerungswahl von nominierten Delegierten und Ersatz- delegierten der Delegiertenversammlung}

7.1 Bestätigungswahl von nominierten DV-Delegierten

Die ÄK stimmt über die Bestätigung der folgenden ordentlichen DV-Delegierten ab:

VEDAG:

Bisher: Schreier Kurt, Gersau

Neu: $\quad$ Widler Josef, Zürich

SMSR:

Bisher: Gauchat Marc-Henri, Sion

Neu: $\quad$ Gusmini Mauro Walter, La Chaux-de-Fonds

Antrag:

Die antragstellenden Organisationen beantragen der $\ddot{A} K$, die aufgeführten ordentlichen DV-Delegierten neu zu bestätigen.

Beschluss:

Die Anträge werden ohne Gegenstimme mit 2 Enthaltungen genehmigt.

7.2 Verlängerungswahl von nominierten DV-Delegierten

In Anwendung von Art. 22 der FMH-Statuten ersuchen die antragstellenden Gesellschaften die ÄK, folgende Mandatsverlängerungen zu genehmigen.
FMPP:

Verlängerung Mandat von Christian Bernath

SFSM:

Verlängerung Mandat Marcus Lütolf

Antrag:

Es wird beantragt, von Art. 22 der FMH-Statuten abzuweichen, damit die DV-Delegierten Bernath und Lütolf ihre Mandate innerhalb der FMH über das Alter von 68 Jahren hinaus bis Mai 2016 weiterführen können.

\section{Beschluss:}

Die Anträge FMPP und SFSM werden mit 4 Gegenstimmen und 7 Enthaltungen genehmigt.

\section{Finanzplanung / Finanzcontrolling}

Anne-Geneviève Bütikofer/GS FMH gibt einen Überblick über die bereits umgesetzten Massnahmen. Dabei geht es um die Zentralisierung des Planungs- und Budgetierungsprozess, die Einführung von neuen Instrumenten gemäss Art. 50 Abs. 2 der Statuten wie z.B. Leistungsverträge, die Umsetzung der vom ZV verabschiedeten neuen Sponsoringstrategie sowie die Evaluation und Auflösung überschüssiger Rückstellungen. Aktuell arbeitet das GS an der Einführung eines Projektplanungs- und -steuerungsinstruments sowie an der Neugestaltung des Risikomanagements. In diesem Zusammenhang werden ebenfalls die Geschäfts- und Arbeitsprozesse evaluiert. Die Revision der Sitzungs- und Spesenentschädigungen für FMHOrgane und Kommissionen wie die Einführung der Ausgabenbremse wurde heute von der ÄK bereits genehmigt. Das Personalreglement wird revidiert und nächstes Jahr in Kraft gesetzt. Weitere geplante Massnahmen sind der Budgetprozess nach Aufgabenprogramm und Projekten, die Analyse der Anlagestrategie sowie die Erstellung eines Anlagereglements.

Ein kritischer Punkt bilden die Leistungsverträge. Im Rahmen der Massnahmen zur finanziellen Stabilisierung der FMH hatte der ZV am 27. August 2013 einstimmig beschlossen, einen Leistungsvertrag mit allen Leistungsempfängern abzuschliessen, die von der FMH mit mehr als CHF 10000 pro Jahr unterstützt werden. Am 8. Mai 2014 hat die ÄK von der Übersicht der Leistungsempfänger, für die ein Leistungsvertrag abgeschlossen werden muss, Kenntnis genommen. Heute geht es darum, dass die ÄK bestimmt, dass die FMH für jegliche finanzielle Unterstützung von mehr als CHF 10000 einen Leitungsvertrag abschliessen muss, insbesondere auch mit der EMH, der SMSR und dem OMCT.

Vincenzo Liguori/TI vertritt in einer ausführlichen Stellungnahme die Ansicht, dass es sich hier um Leistungsverträge handelt, die die ÄK 2011 bereits gutgeheissen hat. Schon 2010 hat die ÄK festgestellt, dass die EMH sich in einer schwierigen Situation befand, weshalb eine Task Force ernannt wurde. Kurz vor 17.00 Uhr über dieses Traktandum einen Beschluss zu fassen, ist unmöglich. 
Über den Antrag des ZV kann nicht mehr abgestimmt werden, da gemäss Art. 11 Abs. 3 der GO ab 17.00 Uhr keine Beschlüsse mehr gefasst werden. Das Geschäft wird für die nächste ÄK traktandiert.

Die übrigen Traktanden 9 (Aufgabenüberprüfung FMH), 10 (Dienstleistungskatalog der FMH) und 11 (Zusammenarbeit FMH - FMH Services) konnten nicht mehr behandelt werden. Die Delegierten haben für Traktandum 12 bereits mit dem Einladungsversand die Berichte aus Zentralvorstand, Generalsekretariat und Abteilungen zugestellt erhalten.

Jürg Schlup/Präsident FMH macht nochmals darauf aufmerksam, dass die Sitzungsunterlagen für die nächste ÄK mit dem neuen IT-Tool zugestellt werden.
Eine entsprechende Dokumentation finden die ÄKDelegierten in den heute verteilten Unterlagen.

Weiter informiert der Präsident, dass das Bundesverwaltungsgericht auf die Beschwerde von $\mathrm{H}+$ gegen die Verordnung über die Anpassung von Tarifstrukturen in der Krankenversicherung aus formalen Gründen nicht eingetreten ist. Der Entscheid auf die Beschwerde von fmCh ist noch ausstehend.

Die nächste ÄK findet am 7. Mai 2015 in Biel statt.

Der Präsident dankt den Delegierten, den Mitgliedern des ZV, der Generalsekretärin sowie allen Mitarbeitenden des Generalsekretariats für die konstruktive und sachliche Sitzungsarbeit. Er wünscht allen eine gute Heimkehr.

\begin{tabular}{|c|c|c|c|}
\hline \multicolumn{4}{|l|}{ Glossar } \\
\hline Abkürzung & Erläuterung & MedBG & Medizinalberufegesetz \\
\hline AGZ & Ärztegesellschaft Kanton Zürich & NAKO & Nationale Konsolidierungsstelle \\
\hline ÄK & Ärztekammer & \multirow[t]{2}{*}{ OMCT } & \multirow{2}{*}{$\begin{array}{l}\text { Ordine dei Medici del Cantone } \\
\text { Ticino }\end{array}$} \\
\hline DRG & Diagnosis Related Groups & & \\
\hline DV & Delegiertenversammlung & SAMW & $\begin{array}{l}\text { Schweizerische Akademie der } \\
\text { medizinischen Wissenschaften }\end{array}$ \\
\hline EPD & Elektronisches Patientendossier & SÄZ & Schweizerische Ärztezeitung \\
\hline GDK & $\begin{array}{l}\text { Schweizerische Konferenz der } \\
\text { kantonalen Gesundheitsdirektoren }\end{array}$ & \multirow{2}{*}{$\begin{array}{l}\text { SGK SR } \\
\text { und NR }\end{array}$} & \multirow{2}{*}{$\begin{array}{l}\text { Kommission für soziale Sicherheit } \\
\text { und Gesundheit Ständerat und } \\
\text { Nationalrat }\end{array}$} \\
\hline \multirow[t]{2}{*}{ eHealth } & \multirow{2}{*}{$\begin{array}{l}\text { Zusammenfassung aller elektro- } \\
\text { nischen Gesundheitsdienste }\end{array}$} & & \\
\hline & & \multirow[t]{2}{*}{ SFSM } & \multirow{2}{*}{$\begin{array}{l}\text { Swiss Federation of Specialties in } \\
\text { Medicine }\end{array}$} \\
\hline eKG & elektronische Krankengeschichte & & \\
\hline EMH & Schweizerische Ärztezeitung AG & \multirow[t]{2}{*}{ SGIM } & \multirow{2}{*}{$\begin{array}{l}\text { Schweizerische Gesellschaft } \\
\text { für Allgemeine Innere Medizin }\end{array}$} \\
\hline \multirow[t]{2}{*}{$\mathrm{fmCh}$} & foederatio medicorum & & \\
\hline & chirurgicorum helvetica & \multirow[t]{2}{*}{ SGMO } & \multirow{2}{*}{$\begin{array}{l}\text { Schweizerische Gesellschaft } \\
\text { für Medizinische Onkologie }\end{array}$} \\
\hline \multirow[t]{2}{*}{ FMPP } & Foederatio Medicorum Psychiatri- & & \\
\hline & corum et Psychotherapeuticorum & \multirow[t]{2}{*}{ SGPP } & \multirow{2}{*}{$\begin{array}{l}\text { Schweizerische Gesellschaft } \\
\text { für Psychiatrie und Psychotherapie }\end{array}$} \\
\hline \multirow[t]{2}{*}{ FMH } & Verbindung der Schweizer & & \\
\hline & Ärztinnen und Ärzte & \multirow[t]{2}{*}{ SMSR } & \multirow{2}{*}{$\begin{array}{l}\text { Société médicale de la } \\
\text { Suisse romande }\end{array}$} \\
\hline GPK & Geschäftsprüfungskommission & & \\
\hline GO & Geschäftsordnung & \multirow[t]{2}{*}{ SIWF } & \multirow{2}{*}{$\begin{array}{l}\text { Schweizerisches Institut für } \\
\text { ärztliche Weiter- und Fortbildung }\end{array}$} \\
\hline GS & Generalsekretärin & & \\
\hline HPC & Health Professional Card & TARMED & $\begin{array}{l}\text { Tarif Medizin für ambulante } \\
\text { Einzelleistungen }\end{array}$ \\
\hline IPI & Institut für Praxisinformatik & \multirow[t]{2}{*}{ VEDAG } & \multirow{2}{*}{$\begin{array}{l}\text { Verband deutschschweizerischer } \\
\text { Ärztegesellschaften }\end{array}$} \\
\hline KHM & Kollegium für Hausarztmedizin & & \\
\hline KKA & $\begin{array}{l}\text { Konferenz der Kantonalen } \\
\text { Ärztegesellschaften }\end{array}$ & VSAO & $\begin{array}{l}\text { Verband Schweizerische Assistenz- } \\
\text { und Oberärzt-/innen }\end{array}$ \\
\hline KVG & Krankenversicherungsgesetz & ZV & Zentralvorstand \\
\hline
\end{tabular}

